\title{
Norois
}

Environnement, aménagement, société

$235 \mid 2015$

La directive cadre sur l'eau à l'échéance 2015. Les

façades océaniques

\section{Transferts de pesticides dans un petit bassin versant viticole des coteaux du Layon : importance des pics lors du ruissellement}

Transport of pesticides in a small vineyard catchment of Layon valley: high contribution of peaks concentrations during runoff

Isabelle La Jeunesse, Audrey Amiot, David Landry, Alain Jadas-Hécart, Pierre-Yves Communal, Aziz Ballouche, Bruno Vitrai et Laurent Mounereau

\section{OpenEdition}

Journals

Édition électronique

URL : https://journals.openedition.org/norois/5618

DOI : $10.4000 /$ norois. 5618

ISBN : 978-2-7535-4775-9

ISSN : $1760-8546$

Éditeur

Presses universitaires de Rennes

Édition imprimée

Date de publication : 15 novembre 2015

Pagination : 67-86

ISBN : 978-2-7535-4771-1

ISSN : 0029-182X

\section{Référence électronique}

Isabelle La Jeunesse, Audrey Amiot, David Landry, Alain Jadas-Hécart, Pierre-Yves Communal, Aziz

Ballouche, Bruno Vitrai et Laurent Mounereau, «Transferts de pesticides dans un petit bassin versant viticole des coteaux du Layon : importance des pics lors du ruissellement », Norois [En ligne], 235 |

2015, mis en ligne le 15 novembre 2017, consulté le 13 janvier 2022. URL : http://

journals.openedition.org/norois/5618; DOI : https://doi.org/10.4000/norois.5618 

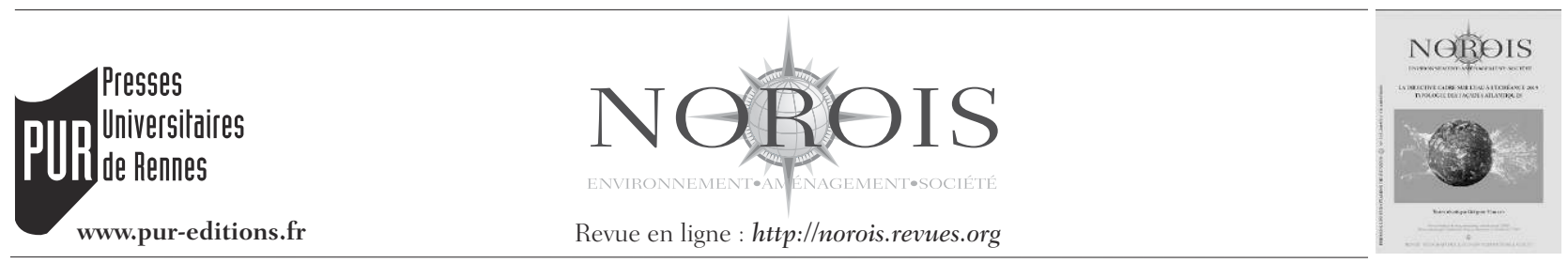

\title{
Transferts de pesticides dans un petit bassin-versant viticole des coteaux du Layon : importance des pics lors du ruissellement
}

\author{
Transport of Pesticides in a Small Vineyard Catchment of Layon Valley: \\ High Contribution of Peaks Concentrations during Runoff
}

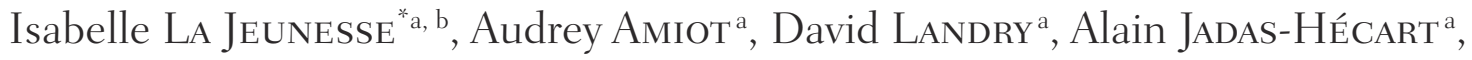 \\ Pierre-Yves Communal ${ }^{\text {a c }}$, Aziz Ballouche ${ }^{a}$, Bruno VITRaI $^{d}$ et Laurent Mounereau ${ }^{d}$
}

\footnotetext{
*Auteur correspondant : (isabelle.lajeunesse@univ-tours.fr)

a URR CNRS 6554 LETG-Angers LEESA, Université d'Angers - 2 bd Lavoisier, 49045 ANGErs Cedex 1, France.

b UMR CNRS 7324 Citeres, Université de Tours, Maison des Sciences de l'Homme - 33 allée Ferdinand-deLesseps, BP 60449, 37204 Tours Cedex 3, France.

c GIRPA, Groupement Interrégional des Produits Agro-pharmaceutiques - 9 av. du Bois l’Abbé, 49071 BeAucouzé, France.

d SAGE Layon-Aubance - 3, Place Joseph-Cousin, 49540 Martigné-Briand, France.
}

Résumé : La Directive européenne Cadre sur l'Eau demande pour 2015 d'atteindre le bon état chimique et écologique des masses d'eaux des États membres. Pour les pesticides, cela revient à s'assurer que les concentrations dans les rivières ne dépassent pas $0,1 \mu \mathrm{g} / \mathrm{L}$ par molécule et $0,5 \mu \mathrm{g} / \mathrm{L}$ pour la somme des concentrations des différentes molécules retrouvées. La reconquête de la qualité du milieu requiert donc de limiter les transferts de pesticides des parcelles agricoles vers les rivières. La viticulture représente $20 \%$ de la consommation nationale en pesticides. Les territoires viticoles sont ainsi souvent sujets à de fortes concentrations en pesticides dans les rivières. La rivière du Layon, soumise à un fort dépassement de la norme DCE vis-à-vis des pesticides, fait l'objet d'un programme d'actions du SAGE Layon-Aubance-Louet. Son objectif est d'atteindre, pour la somme des concentrations en pesticides, $1 \mu \mathrm{g} / \mathrm{L}$ en 2018 et $0,5 \mu \mathrm{g} / \mathrm{L}$ à l'horizon 2027. Dans ce contexte, une des actions du SAGE consiste à comprendre les modalités de transferts de pesticides dans les sous bassins-versants notamment par le suivi des pics de pesticides en provenance d'un petit bassin-versant exclusivement viticole des coteaux du Layon, instrumenté par l'Université d'Angers. Les résultats montrent que les pics de concentrations en pesticides les plus importants se situent lors des événements pluvieux les plus rapprochés des dates d'application de la molécule sur les vignes. Les pics de concentrations sont également en phase avec les maximums de concentrations en matières en suspension. Aussi, bien que l'enherbement sur les parcelles contribue à limiter l'érosion notamment en favorisant la stabilité du sol, il ne suffit pas à stopper les transferts de pesticides.

\begin{abstract}
The European Water Framework Directive application requires achieving, in 2015, not only good ecological status but also good chemical status for natural waters in Member States. For pesticides, this means ensuring that concentrations in rivers do not exceed 0.1 $\mu \mathrm{g} / \mathrm{L}$ per molecule and $0,5 \mu \mathrm{g} / \mathrm{L}$ for the sum of the concentrations of the different molecules found. The treatment of vineyards represents $20 \%$ of the national consumption of pesticides. The wine territories are often subject to high concentrations of pesticides in rivers. Thus, the recovery of the quality of the environment requires action programs to minimize the amount of pesticides filtering into the river. The river Layon is therefore subject to a program of actions led by the local water committee, the SAGE Layon-Aubance-Louet. Its goal is to ensure pesticide concentrations are reduced to $1 \mu \mathrm{g} / \mathrm{L}$ in 2018 and $0,5 \mu \mathrm{g} / \mathrm{L}$ in 2027. In this context, one of the actions of the SAGE, with the assistance of the University of Angers, addresses the study of peaks in pesticide concentrations during runoff events in a small catchment covered by vineyards. Results demonstrate first a peak intensity of pesticides in runoff waters in relation with the date of application with a decrease of concentrations during time after the treatment and second a relation between peaks of suspended particulate matter (SPM)
\end{abstract}


and pesticides. Transfer of pesticides in this catchment is strongly linked to runoff. Thus, the percentage of surface remained un-weeded, if it improves the stability of soil permitting to decrease erosion, is not sufficient to stop the transfer.

Mots clés : DCE, politique de l'eau - gestion des ressources en eau - pollution - ruissellement - bassin-versant - vignoble - Union européenne

Keywords: EWFD - water policy - water management - pollution - runoff - small catchment - vineyard - European Union

\section{INTRODUCTION}

L'utilisation des pesticides est une histoire ancienne et remonterait à l'Antiquité. Toutefois, les progrès de la chimie minérale puis organique au sortir de la $2^{\mathrm{e}}$ guerre mondiale sont concomitants au développement de l'utilisation des décennies passées (ORP, 2015). Les pesticides sont rendus quasiment indispensables aux pratiques agricoles et à l'assurance d'une production de qualité et surtout à l'assurance d'un rendement.

On dénombre aujourd'hui plus de 480 substances commercialisées et 2282 spécialités commerciales (Petit, 2014). Les pays développés sont, aujourd'hui encore et ce malgré l'augmentation de la consommation des pays en développement, les plus consommateurs de pesticides. Ce sont 5,4 kg de molécules actives qui sont épandus en moyenne par hectare de surface agricole cultivée et par an (Aubertot et al., 2005).

De nombreuses études scientifiques mettent régulièrement en évidence l'impact de la pollution des eaux par des pesticides (Kohler et Triebskorn, 2013; Schwarzenbach et al., 2006). Un auteur de la prestigieuse revue Nature (Vörösmarty et al., 2010) va même jusqu'à estimer à près de $80 \%$ la proportion de la population mondiale qui serait concernée par des ressources en eau dégradées par la présence de divers contaminants et plus particulièrement par les pesticides. En effet, l'utilisation mondiale massive de pesticides fait de cette catégorie d'intrants un des polluants majeurs de l'environnement. Or les pesticides sont également reconnus comme perturbateurs des activités biologiques, bactériennes (Imfeld et Vuilleumier, 2012) et biogéochimiques (Miguens et al., 2007) du sol et de la flore (Andresen et al., 2012). Parallèlement aux problématiques écologiques, des risques sanitaires réels sont aujourd'hui établis (Mostafalou et Abdollahi, 2013) et on reconnaît des relations de causes à effets entre de fortes expositions à certains pesticides et l'apparition de cancers (Bassil et al., 2007) ou de la maladie de Parkinson (Fitzmaurice et al., 2013).

La France, parce qu'elle est un pays fortement agricole notamment pour la production de céréales, reste le premier pays consommateur de pesticides en Europe et le $4^{\mathrm{e}}$ au niveau mondial derrière les Etats-Unis, le Brésil et le Japon et ce malgré une baisse significative des consommations ces dernières années (Aubertot et al., 2005). Environ $90 \%$ des 78600 tonnes des substances actives vendues en 2008 en France l'ont été pour des usages agricoles (Gatignol et Étienne, 2010). La vigne, alors qu'elle représente $3 \%$ de la Surface Agricole Utile française, représente $20 \%$ de la consommation française totale de pesticides (Aubertot et al., 2005). Parallèlement, la contamination des cours d'eau en pesticides en France est pour ainsi dire généralisée. D'après le bilan annuel de l'observatoire des statistiques du ministère de l'écologie, du développement durable et de l'énergie, seuls $11 \%$ des prélèvements d'eau superficiel et souterrain ne seraient pas contaminés. Ils concerneraient en fait des régions peu agricoles ou avec une agriculture peu intensive, notamment le quart sud-est et l'Auvergne (MEDDE, 2015).

La Directive Cadre sur l'Eau de la Commission Européenne de 2000 (DCE2000/60) et à laquelle la France a largement contribué à la mise en place, a été pensée dans l'objectif de ne plus retrouver de pesticides dans les rivières. Pour autant, 15 ans après, tous les territoires concernés par la DCE n'ont pas atteint le bon état écologique de leurs masses d'eau.

Dans le Val de Loire, inscrit depuis 2003 au patrimoine mondial de l'Unesco pour ses paysages culturels vivants de vignes et de vins, la rivière Layon connaît des dépassements systématiques des normes de la DCE vis-à-vis des pesticides. Aussi les gestionnaires du territoire sont face à des enjeux économiques et environnementaux qui consistent à 
promouvoir la production de vins prestigieux dans un site inscrit au patrimoine mondial dans le respect des normes environnementales. Pourtant, le Layon n'est pas le seul territoire à utiliser des pesticides sur son vignoble. Y aurait-il une spécificité du Layon? Si oui, pour quels critères? Les caractéristiques du sol? Climatiques? Des pratiques agricoles?

Afin de remédier aux contaminations par les pesticides des milieux aquatiques, les modalités de transferts depuis les petits bassins-versants viticoles doivent être connues (Oliver et al., 2012). L'objectif de l'étude commandée par le SAGE Layon-Aubance-Louet dans le cadre du programme d'actions du Contrat Régional Bassin Versant vise ainsi à mettre en évidence les transferts de pesticides lors du ruissellement dans les bassins-versants viticoles.

L'objectif des prélèvements des eaux de ruissellement sur un petit bassin-versant viticole présenté dans cet article est de suivre en temps réel des concentrations en pesticides à l'exutoire du bassinversant. En effet les analyses réalisées par le réseau de surveillance de la qualité des eaux de la rivière sont ponctuelles et ne montrent que le bruit de fond des polluants sans être représentatives de la gamme d'évolution des concentrations réelles qui sont susceptibles de varier lors des événements pluvieux. Ces valeurs de concentrations en pesticides des eaux du Layon issues de prélèvements instantanés dans la rivière ne reflètent donc pas l'état de contamination de la rivière, ni les apports importants issus des affluents lors des crues et ce notamment pour l'herbicide glyphosate (Daouk et al., 2013), toujours présent dans les eaux du Layon. Par ailleurs, des échantillons moyens sous-estiment généralement les concentrations en pesticides et les flux transférés par les rivières (Rabiet et al., 2010). Ainsi le suivi des pesticides sur ce bassinversant a pour objectif de mettre en évidence les pics de concentrations lors du ruissellement. En effet, les pics, parce qu'ils représentent les plus fortes concentrations, peuvent être déterminants pour les compartiments vivants des cours d'eau car ils peuvent correspondre à de plus forts taux de toxicité. Aussi, contrairement à des échantillons moyens, l'étude des pics permet d'observer s'il y a des comportements similaires ou des différences en fonction des molécules transférées.
Les processus d'érosion de surface sont régis par les processus de désintégration des agrégats sous l'action de l'eau (Le Bissonnais et Singer, 1993; Barthès et Roose, 2002; Ramos et al., 2003). Les particules érodées et transférées dans les parcelles agricoles sont généralement celles inférieures à $250 \mu \mathrm{m}$ (Panuska et al., 2008). Ainsi, selon Saygina et al., 2012, l'étude de la sensibilité des sols à produire des particules fines serait un meilleur moyen d'estimer leur sensibilité à l'érosion. Cette fraction fine peut avoir un rôle important dans le transfert des pesticides et notamment du glyphosate. En effet, le glyphosate est communément reconnu pour être fortement adsorbé sur les limons fins et les argiles (Damonte et al., 2007; Glass, 1987). Or les mécanismes de désintégration des agrégats sont intrinsèquement liés à la stabilité des agrégats (Le Bissonnais et Souder, 1995; Le Bissonnais, 1996). C'est pourquoi, dans cette étude, l'hypothèse faite est que les matières en suspension (MES), majoritairement constituées de particules inférieurs à $250 \mu \mathrm{m}$, peuvent être assimilées à la fraction instable fine des sols (Kemper et Rosenau, 1986). Parallèlement à l'étude des modalités de transferts a donc été menée une étude sur la fraction fine du sol du bassin-versant. Afin de comprendre la dynamique du transfert, il est nécessaire de comprendre comment les pesticides sont retenus à la surface puis comment ils sont véhiculés par l'eau et les MES. Sont présentés ici deux aspects de l'étude : (i) les quantités de résidus de pesticides et de MES exportées lors d'épisodes de ruissellement de l'année hydrologique 2012, (ii) la stabilité structurale en fonction de l'état de surface du sol (enherbé, désherbé mécaniquement ou chimiquement).

Dans cet article, après une présentation du site d'étude et des objectifs du SAGE Layon-AubanceLouet, la station de mesures et les protocoles d'échantillonnage des eaux de ruissellement et des sols du bassin-versant instrumenté sont décrits. Les résultats des pics de pesticides lors des événements pluvieux ayant donné lieu à du ruissellement lors de l'année hydrologique 2012 sont présentés et discutés au regard de la composition des sols, de l'érosion, des quantités appliquées et des objectifs de qualité environnementale. 


\section{LA POLLUTION DES EAUX PAR LES PESTICIDES}

La pollution par des pesticides est principalement perçue au travers de leur présence dans les eaux. Cependant, la majeure partie des polluants transitent par les sols où leur comportement va conditionner la manifestation de leur caractère polluant en fonction de processus de rétention et de stabilisation, de transformation, ainsi que des phénomènes de transfert (figure 1 et figure 2 - planche IV). Le sol occupe donc une position centrale dans la régulation des pollutions (Calvet et al., 2005) avec un double rôle d'épuration et de stockage des polluants propre à chaque molécule. Il participe ainsi à leur élimination comme au retard ou à la diminution des transferts.

\section{Rétention des pesticides par les constituants des sols : l'adsorption}

Parmi les caractéristiques des sols, la teneur en matières organiques est l'un des paramètres les plus corrélés avec les coefficients d'adsorption (Barriuso et Calvet, 1992) pour de nombreuses molécules, mais intervient également la valeur du $\mathrm{pH}$, la teneur en argile, la teneur en fractions fines et la présence d'oxyhydroxydes métalliques. Les processus de rétention des pesticides dans le sol réduisent leur mobilité et diminuent ainsi, au moins temporairement, leur transfert vers l'eau. Cette rétention évolue néanmoins dans le temps et peut devenir à peu près irréversible jusqu'à créer des résidus liés, non extractibles, dont on ne connaît ni la nature chimique exacte, ni la capacité de désorption ultérieure.

\section{Dégradation des pesticides dans les sols : la persistance}

Le processus de dégradation peut être un facteur de dépollution majeur des compartiments environnementaux contaminés par les pesticides, s'il aboutit toutefois à une minéralisation totale. La dégradation du polluant s'accompagne de l'apparition de métabolites, avec un changement de la structure chimique, provoquant des modifications de leur toxicité et de leur comportement dans les sols par rapport à celui de la molécule mère (Benoît, 1994). Ces processus de dégradation dépendent de la stabilité chimique de la molécule et de facteurs biologiques (microorganismes du sol dits microflore) et abiotiques (température et humidité).

La rétention par le sol et la persistance d'un pesticide ne sont en fait pas des phénomènes indépendants : la rétention conditionne la disponibilité des pesticides pour leur dégradation (Barriuso et al., 1996). En pratique, c'est donc le couple rétentiondégradation qui détermine la mobilité des pesticides.

\section{Mobilité des pesticides : transport par ruissellement, infiltration et volatilisation}

La part du polluant la plus mobile est localisée dans les phases liquide et gazeuse du sol. Elle constitue la fraction disponible pour être dégradée par les micro-organismes du sol et pour être entraînée par les eaux.

Le ruissellement est en grande partie responsable du départ des produits vers les eaux superficielles. Il est lié à la conjonction de plusieurs facteurs favorables à sa mise en place tels que la topographie, l'intensité des précipitations, la nature et la structure du sol, la nature du couvert végétal et l'intervalle de temps qui sépare l'application du pesticide de la première pluie. Le transfert des pesticides par ruissellement peut s'opérer soit en solution, c'est-àdire sous forme dissoute, soit par l'intermédiaire des matières en suspension dans l'eau (Dur et al., 1998).

Le transfert vertical des pesticides dans les sols, dit encore lixiviation, est à l'origine de la contamination de la nappe souterraine.

La volatilisation est le passage du pesticide du sol, de l'eau ou des végétaux vers l'atmosphère. Ce phénomène dépend des propriétés physico-chimiques des molécules (constante de volatilisation ou constante de Henry), des conditions climatiques (vent, température de l'air) et des pratiques agricoles (incorporation ou non des produits dans les couches superficielles des sols).

La présente étude se focalise sur l'évolution des concentrations de pesticides et matières en suspension (MES) lors du ruissellement, sans réalisation de bilan de matière à l'échelle du bassin-versant. 


\section{Site D'ÉTUde}

\section{Objectifs de reconquête de la qualité des eaux du Layon du SAGE Layon- Aubance-Louet}

La rivière le Layon prend sa source dans le département des Deux-Sèvres et traverse le département de Maine-et-Loire d'est en ouest avant de se jeter dans la Loire (figure 3). Dans le bassin-versant du Layon et de l'Aubance, l'adduction en eau potable, comprenant les usages industriels très minoritaires, représente $20 \%$. Les $80 \%$ restant sont représen- tés par l'irrigation. L'eau potable produite dans le SAGE Layon-Aubance-Louet provient uniquement de la ressource en eau souterraine. L'eau d'irrigation provient, en proportion égale, d'eau superficielle et souterraine. Les prélèvements annuels représentaient 8,4 millions de $\mathrm{m}^{3}$ en 2011 (SIGES Pays-dela-Loire, 2015). La problématique de qualité des eaux superficielles concerne donc essentiellement le milieu naturel. Or cette rivière est parfois citée localement comme la rivière la plus polluée de France (Wajdzik, 2008). Le Layon souffre, comme mis en évidence par les évaluations de l'IBGN (Indice

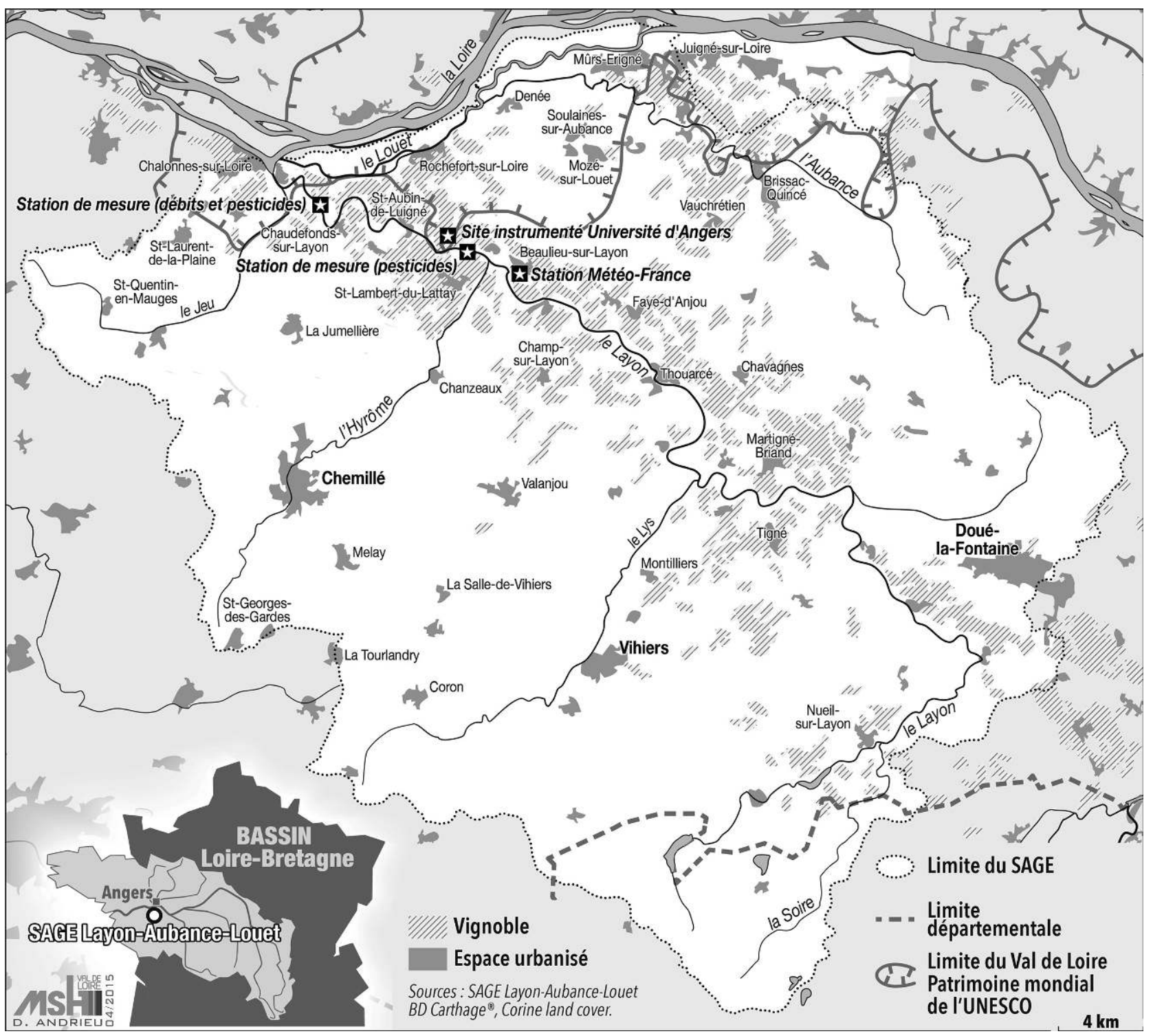

Figure 3 : Carte du SAGE Layon-Aubance-Louet

Location map of the SAGE Layon-Aubance-Louet 
Biologique Global Normalisé), d'une forte dégradation écologique (SAGE, 2004; SAGE, 2013) en plus d'un état chimique global médiocre depuis de nombreuses années (Contrat Territorial, 2010).

Les concentrations moyennes en pesticides dans le Layon dépassent les seuils de la DCE (tableau 1). Les données présentées sont issues d'analyses réalisées par la CREPEPP (Conférence REgionale EcoPhyto En PluriPartenariat) à la station de La Pierre Saint-Maurille à Chaudefonds-surLayon (figure 3). Les données de débit sont celles mesurées à la station de Saint-Lambert-du-Lattay (figure 3) au Pont de Bézigon (source: Banque
Hydro). Les échantillons prélevés dans les eaux du Layon de 1996 à 2007 (Abdoul-Madjidi, 2008; La Jeunesse et al., 2010) et de 2010 à 2013 (tableau 2) ne montrent pas de corrélation systématique entre le débit, le nombre de molécules détectées et la somme des concentrations en pesticides. Par contre, le nombre de molécules détectées augmente de 2010 à 2013 (tableau 1 et 2). Parmi ces 4 années, c'est l'année 2011, année la plus sèche en termes de précipitations (figure 4) et en termes de débit (tableau 3) qui présente la situation la plus dégradée du point de vue des concentrations en pesticides. Cette situation pourrait être expliquée par la sévé-

\begin{tabular}{|l|c|c|c|c|}
\cline { 2 - 5 } \multicolumn{1}{c|}{} & 2010 & 2011 & 2012 & 2013 \\
\hline Fréquence de dépassement de $0,5 \mu \mathrm{g} / \mathrm{l}(\%)$ & 88,2 & 94,7 & 88,9 & 94,4 \\
\hline Fréquence de dépassement de $1 \mu \mathrm{g} / \mathrm{l}(\%)$ & 52,9 & 73,7 & 66,7 & 61,1 \\
\hline Nb maximum de molécules détectées & 14 & 28 & 25 & 33 \\
\hline
\end{tabular}

Tableau 1 : Nombre de molécules détectées et fréquence (en pourcentage) de dépassement du seuil légal des concentrations en pesticides à Chaudefonds-sur-Layon de 2010 à 2013 (CREPPEP) from 2010 to 2013

Number of molecules detected and percentage of exceeding legal thresholds of pesticides concentrations at Chaudefonds-sur-Layon

\begin{tabular}{|c|c|c|c|c|c|}
\hline & & 2010 & 2011 & 2012 & 2013 \\
\hline Débit & Module $\left(\mathrm{m}^{3} / \mathrm{s}\right)$ & 3,4 & 1,5 & 4,8 & 6,1 \\
\hline \multirow{4}{*}{ Nombre de molécules détectées } & Moyenne & 7 & 14 & 14 & 17 \\
\hline & Minimum & 2 & 4 & 4 & 9 \\
\hline & Percentile 90 & 12 & 24 & 20 & 27 \\
\hline & Maximum & 14 & 28 & 25 & 33 \\
\hline \multirow{4}{*}{ Somme des concentrations en pesticides $(\mu \mathrm{g} / \mathrm{L})$} & Moyenne & 1,05 & 2,05 & 1,17 & 1,21 \\
\hline & Minimum & 0,25 & 0,47 & 0,42 & 0,43 \\
\hline & Percentile 90 & 1,62 & 4,36 & 1,73 & 1,89 \\
\hline & Maximum & 1,73 & 5,55 & 1,84 & 2,61 \\
\hline
\end{tabular}

Tableau 2 : Nombre de molécules détectées dans les eaux du Layon à Chaudefonds-sur-Layon de 2010 à 2013 (CREPPEP, Banque Hydro) Number of molecules detected in the Layon river at Chaudefonds-sur-Layon from 2010 to 2013

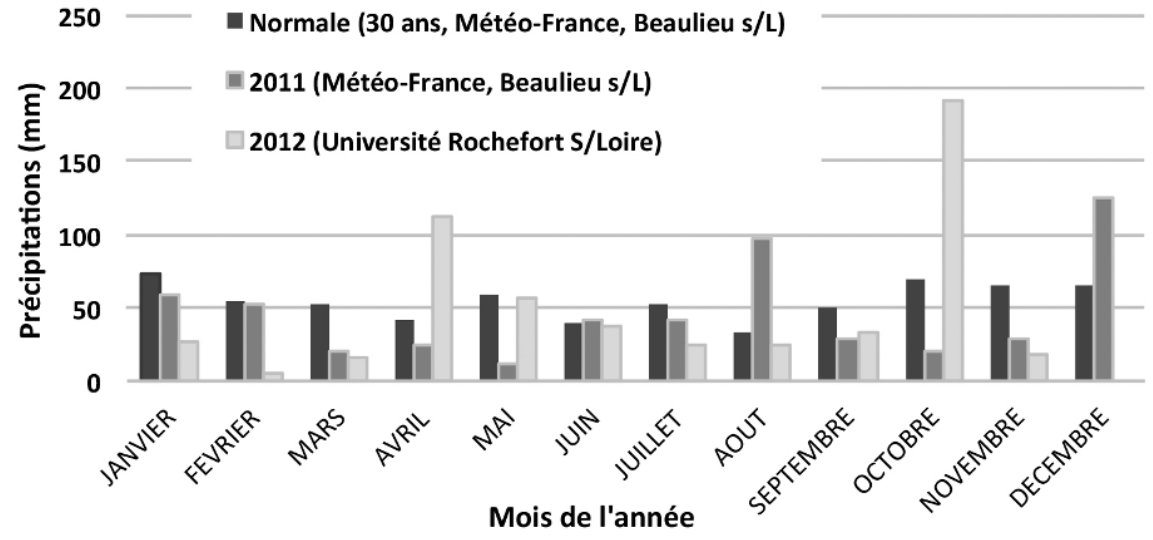

Figure 4 : Précipitations des mois de l'année 2012 de janvier à novembre sur le site instrumenté (pas de mesures pour décembre 2012) comparativement à la normale (moyenne sur 30 ans) et à l'année 2011 à la station Météo-France de Beaulieu-sur-Layon Monthly precipitation totals from January to November 2012 from the instrumented site (no measure for December 2012) compared to 30 year average situation, and compared to 2011 (measures at Meteo-France sation at Beaulieu-sur-Layon) 


\begin{tabular}{|c|c|c|c|c|}
\hline Débits mensuels & 2010 & 2011 & 2012 & 2013 \\
\hline Janvier & 11,7 & 5,3 & 4,7 & 12,8 \\
\hline Février & 10,5 & 3,1 & 1,7 & 20,5 \\
\hline Mars & 7,7 & 2,3 & 0,6 & 11,7 \\
\hline Avril & 4,9 & 0,8 & 4,0 & 8,7 \\
\hline Mai & 1,1 & 0,5 & 5,3 & 6,5 \\
\hline Juin & 0,7 & 0,3 & 0,8 & 2,2 \\
\hline Juillet & 0,1 & 0,1 & 0,4 & 0,7 \\
\hline Août & 0,1 & 0,2 & 0,1 & 0,2 \\
\hline Septembre & 0,1 & 0,1 & 0,1 & 0,3 \\
\hline Octobre & 0,2 & 0,1 & 11,9 & 0,9 \\
\hline Novembre & 0,5 & 0,4 & 8,3 & 3,6 \\
\hline Décembre & 3,9 & 5,2 & 19,2 & 6,7 \\
\hline Module & 3,5 & 1,5 & 4,8 & 6,2 \\
\hline
\end{tabular}

Tableau 3 : Débits mensuels du Layon à Chaudefonds-sur-Layon de 2010 à $2013\left(\mathrm{~m}^{3} / \mathrm{s}\right)$ (Banque Hydro)

Monthly flows of the Layon river at Chaudefonds-surLayon from 2010 to $2013\left(\mathrm{~m}^{3} / \mathrm{s}\right)$

rité de la période d'étiage. Pourtant, si certes 2011 est une année sèche comparativement aux autres (figure 4), on peut constater que les fréquences de dépassement de la norme $0,5 \mu \mathrm{g} / \mathrm{L}$ (objectif à 2027 du SAGE Layon-Aubance, Contrat territorial, 2015) ne sont pas supérieures à 2013, année humide comme en témoigne le module de la rivière (tableau 2). En effet, 2011 et 2013 ont toutes deux une valeur de $94 \%$ de fréquence de dépassement du seuil à $0,5 \mu \mathrm{g} / \mathrm{L}$ (tableau 1 ). Aussi, le nombre de molécules détecté est plus important en 2013 avec 33 molécules contre 28 détectées dans la rivière en 2011 (tableau 1). Entre 1996 et 2007, une étude sur le Layon a également montré un taux de quantification de $100 \%$ pour la molécule herbicide le glyphosate et son métabolite l'AMPA (acide aminométhylphosphonique) ainsi que pour le fongicide le diméthomorphe (Abdoul-Madjidi, 2008) (tableau 4).

Un objectif de concentrations en pesticides dans les eaux du Layon inférieures à $1 \mu \mathrm{g} / \mathrm{L}$ pour 2018 demeure un objectif ambitieux face à ces fréquences de dépassement importantes aussi bien pour une année sèche (74 \% en 2011) que pour une année humide (61\% en 2013). Face à ce constat, un des objectifs principaux du SAGE Layon-AubanceLouet est de reconquérir la qualité des eaux du Layon vis-à-vis des pesticides. Le SAGE est un outil de planification à l'échelle des bassins-versants du Layon, de l'Aubance et du Louet (figure 3) devant s'inscrire dans les objectifs du Schéma Directeur

\begin{tabular}{|c|c|}
\hline Molécule & $\begin{array}{c}\text { Présence de la molécule dépas- } \\
\text { sant le seuil } 0,1 \mu \mathrm{g} / \mathrm{L}\end{array}$ \\
\hline AMPA & 100 \\
\hline Glyphosate & 100 \\
\hline Diméthomorphe & 100 \\
\hline Carbendazime & 90 \\
\hline Atrazine déisopropyl & 81 \\
\hline Aminotriazole & 80 \\
\hline Norflurazone & 80 \\
\hline Diuron & 74 \\
\hline Therbuthylazine & 61 \\
\hline Pyriméthanil & 54 \\
\hline
\end{tabular}

Tableau 4 : Taux de quantification des molécules retrouvées dans les prélèvements de 1996 à 2007 dans le Layon à Saint-Lambertdu-Lattay (Abdoul Madjidi, 2008)

Quantification rates of molecules detected in water samples from 1996 to 2007 from the Layon river at Saint-Lambertdu-Lattay

d'Aménagement et de Gestion des Eaux (SDAGE) Loire-Bretagne (figure 3), plan de gestion du district hydrographique demandé par la DCE. Pour ce faire, la Commission Locale de l'Eau (CLE) composée d'élus, de représentants de l'Etat, de collectivités et d'usagers de l'eau organise et gère l'ensemble de la procédure d'élaboration, de révision, de consultation, de mise en œuvre et de suivi du SAGE. Elle constitue également un lieu privilégié de concertation, de débat, de mobilisation et de prise de décision.

Pour les eaux superficielles, les objectifs de qualité du SAGE (SAGE, 2013) sont : (i) atteindre le bon état chimique des eaux, (ii) atteindre $1 \mu \mathrm{g} / \mathrm{L}$ pour la somme des substances actives en 2018 et (iii) atteindre $0,5 \mu \mathrm{g} / \mathrm{L}$ en 2027 en percentile 90 (valeur maximale mesurée et non dépassée par $90 \%$ des mesures effectuées).

La stratégie élaborée par la CLE du SAGE LayonAubance-Louet s'appuie sur deux axes de travail (1) la réduction du recours aux pesticides pour les différents usages (agricoles, viticoles, particuliers, collectivités et gestionnaires d'infrastructures), (2) la limitation des transferts des pesticides vers les milieux. Cette stratégie a été élaborée en cohérence avec les objectifs de réduire de $50 \%$ les quantités de pesticides utilisées en France et relevant du plan Ecophyto 2018.

Parallèlement aux objectifs de limitation des applications de pesticides, le SAGE a souhaité connaître 
les modalités de transferts de pesticides dans les sous-bassins versants viticoles du Layon, et notamment identifier l'ampleur des pics de pesticides gagnant la rivière. Le site d'étude est un petit bassin-versant instrumenté par le laboratoire CNRS LETG-Angers de l'université d'Angers dans les coteaux du Layon et plus précisément sur la commune de Rochefort-sur-Loire, commune relevant de la vallée de la Loire inscrite au Patrimoine Mondial de l'Unesco et lieu de production du prestigieux vin liquoreux coteau du Layon Quarts-de-Chaume et Chaume.

\section{Delimitation du bassin-versant hydrologique}

Le site d'étude est un petit bassin-versant viticole de 2,2 ha (figure 5, Guérin, 2008; Rakotoarisoa, 2014). La délimitation topographique est issue d'un traitement sous ArcGIS de 2083 points d'altitudes levés lors d'une campagne de nivellement au théodolite électronique réalisée en 2009 et ayant permis la réalisation du Modèle Numérique de Terrain (MNT, Guérin, 2008; Rakotoarisoa, 2014). La validation du bassin-versant hydrographique a été réalisée lors de plusieurs événements pluvieux où il a été constaté que le chemin en haut des parcelles et les rangs de vignes occasionnent des directions d'écoulement qui modifient significativement les limites du bassin-versant (figure 5, Guérin, 2008; Rakotoarisoa, 2014). La ligne de plus grande pente est de $220 \mathrm{~m}$ environ et définit grossièrement 3 zones avec des ruptures de pente (figure 6 - planche $\mathbf{V}$ ) : haute de pente $(\mathrm{H})$, milieu de pente $(\mathrm{M})$ et bas de pente (B) où se trouvent des terrasses fortement pentues pour la parcelle viticole A (figure 5).

Dans ce site le phénomène dominant de transfert d'eau et de matière est le ruissellement et l'érosion de surface (Rakotoarisoa, 2014; Goyard, 2014). Ce ruissellement se compose d'eaux fortement chargées en MES pouvant potentiellement contenir des résidus de pesticides.

\section{Échantillonnage et analyse des sols}

Les parcelles reposent sur une base lithologique datant du Carbonifère (Namurien). Ces formations primaires - schistes, grès, conglomérats - appartiennent au Sillon houiller de la Basse-Loire (BRGM
- carte géologique au 1/50 000 n 484). Les sols proviennent de l'altération des roches sous-jacentes. Le substratum schisteux affleure à plusieurs endroits des parcelles de milieu et bas de pente. Quelques éléments remontent à la surface à cause du travail du sol, il s'agit de la couche de conglomérats qui repose sur les schistes. Cette couche de conglomérats alimente les sols en éléments grossiers.

Les échantillons de sols ont été prélevés le 4 octobre 2012 dans les inter-rangs de la surface du sol de haut de pente (HP), milieu de pente (MD) et bas de pente (BP) des parties enherbée (E), désherbée mécaniquement ou chimiquement (D). Environ 50 prélèvements (à l'aide d'une tarière à main) ont été répartis sur chaque zone, homogénéisés, tamisés à $2 \mathrm{~mm}$ puis séchés à l'air avant d'être analysés par le laboratoire SAS.

Pour l'étude de la stabilité structurale, les sols ont été échantillonnés à l'aide d'une bèche sur une profondeur 0-20 cm. 25 réplicats ont été réalisés par état de surface du sol et pour les 3 zones de pente. Ces 25 réplicats ont été mélangés, afin d'obtenir un échantillon moyen représentatif. Ces échantillons ont ensuite été séchés à l'air puis tamisés à 2-5 mm, homogénéisés puis analysés.

Les analyses réalisées sont la granulométrie (AFNOR X 31-107) sur un CEC metson (NF X 31,130), les teneurs en matières organiques (MO) (NF ISO 14235), le pH eau (NF ISO 10390).

La fraction fine est obtenue par désagrégation mécanique des sols. Les échantillons de sols ont été séchés à l'air puis tamisés entre 2 et $5 \mathrm{~mm}$.

\section{Échantillonnage des eaux issues du ruissellement}

La station de mesures pour l'échantillonnage des eaux de ruissellement est composée d'un canal jaugeur de type Venturi, d'un canal d'approche pour faciliter la mesure de la hauteur d'eau en limitant les mouvements liés à l'écoulement de l'eau, d'un limnimètre à pression, d'un pluviomètre à augets basculeurs, d'un préleveur automatique de 24 flacons d'1/2 litre, d'une station d'acquisition de données logosens OTTC, d'un logiciel de traitement de données Hydras OTTC, d'un modem pour prévenir le déclenchement du préleveur automatique assujetti à la montée d'eau dans le canal jaugeur et d'une cabane de protection de la station d'acquisition, du 
Figure 5 : Courbes de niveau issues du Modèle Numérique de Terrain digital terrain mode

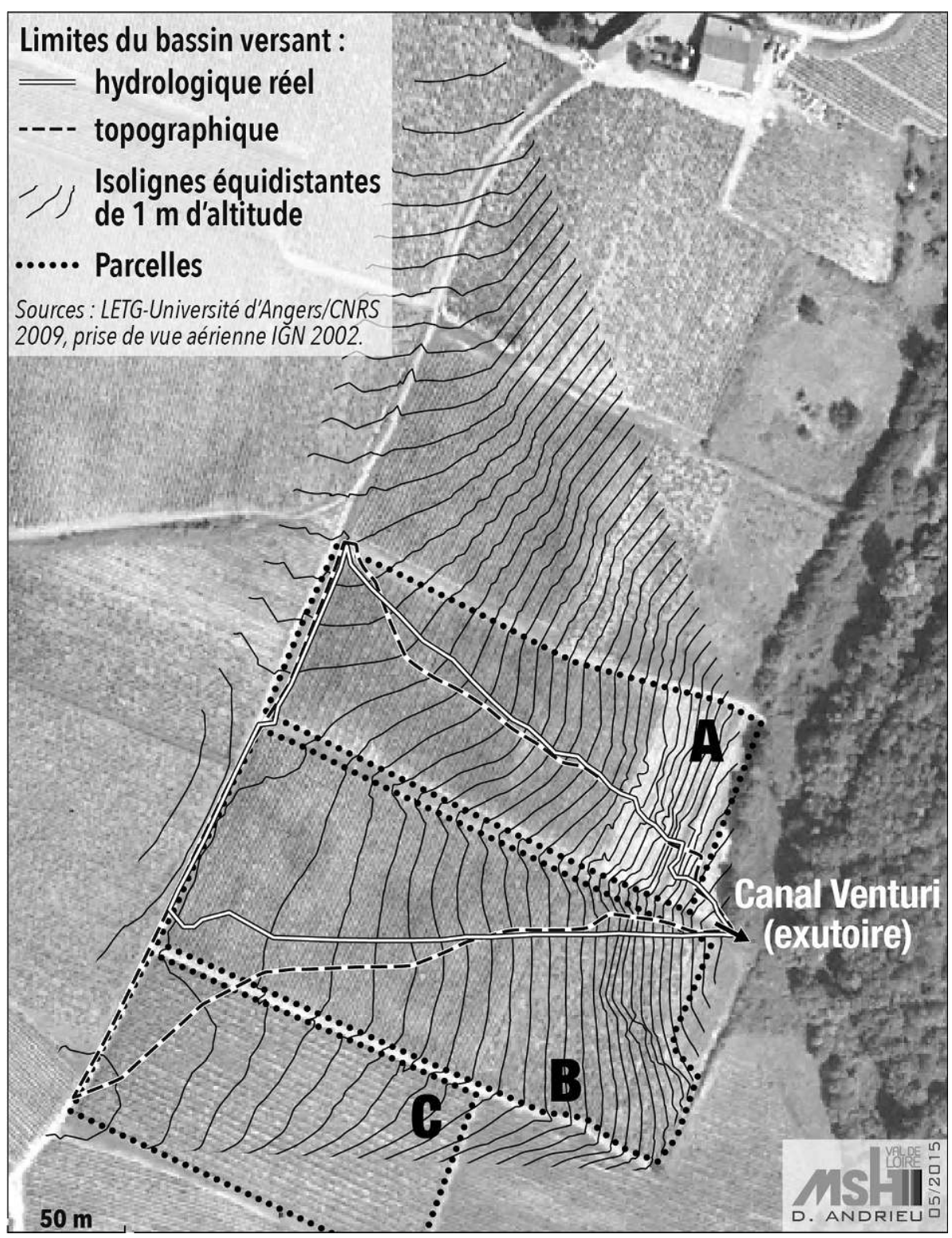

préleveur automatique et de la mise sous pression du limnimètre. Le canal jaugeur Venturi est situé à l'exutoire du bassin-versant (figure 6 - planche V), point de topographie le plus bas où se concentrent les eaux de ruissellement. L'objectif de ce canal est de permettre d'établir une estimation du débit en fonction d'une hauteur d'eau dans le canal.

L'objectif étant de suivre l'évolution des concentrations en pesticides lors des crues et d'identifier les pics lors du ruissellement, le pas de temps de prélèvement le plus court est recherché. Ainsi, lorsqu'une crue génère du ruissellement, la montée d'eau dans le canal déclenche le préleveur auto- matique et le modem envoie un message d'alerte à l'équipe de suivi. Un prélèvement est effectué en 1 minute or il faut 2 prélèvements pour réaliser un échantillon : 1 prélèvement pour nettoyer la chambre de prélèvement et éviter les contaminations avec le prélèvement précédent et 1 prélèvement pour constituer l'échantillon. L'intervalle de 2 minutes est ainsi le plus court possible pour la station de mesures en place. Le préleveur automatique comporte 24 échantillons. Ainsi, si la crue dure plus de 48 minutes, les prélèvements sont effectués manuellement. 


\section{Analyses des MES et des pesticides}

Les échantillons collectés, remontés manuellement depuis la station de mesures située en bas des parcelles viticoles, sont conservés à $4{ }^{\circ} \mathrm{C}$ durant les analyses. Ils sont filtrés à $0,45 \mu \mathrm{m}$ pour l'analyse des MES. La quantité de MES collectée par crue ne permet malheureusement pas de mesurer les concentrations en pesticides adsorbés sur les MES pour les multi-résidus, ni pour le glyphosate et l'AMPA pour lesquels l'extraction est impossible en l'état actuel des connaissances (Aparicio et al., 2013). Ainsi, le filtrat est récupéré pour l'analyse du glyphosate et de l'AMPA au laboratoire LETGAngers. La méthode d'analyse repose sur une dérivation pré-colonne du glyphosate et de l'AMPA par le 9- fluorenylmethoxycarbonyl-chloride (FMOC), après décomplexation des deux composés. L'analyse des dérivés est réalisée par chromatographie liquide couplée à un spectro-fluorimètre. Le seuil de quantification pour la méthode est de $0,1 \mu \mathrm{g} . \mathrm{L}^{-1}$ pour le glyphosate et l'AMPA.

Les analyses des autres molécules sont réalisées par le laboratoire GIRPA associé au laboratoire LETG. La méthode repose sur une extraction des résidus de produits phytosanitaires par partages liquide/liquide avec un mélange dichlorométhane/ acétate d'éthyle. Les extraits organiques sont combi- nés, déshydratés, puis évaporés sous vide. L’analyse est réalisée en chromatographie liquide ultra performance, sur colonne octadécyle, couplée à un détecteur de masse en tandem de type triple quadripôle (LC/ESI/MS/MS) et/ou par chromatographie en phase gazeuse sur colonne capillaire apolaire couplée à un détecteur de masse en tandem de type trappe d'ions (GC/IT/MS/MS) ou liquide en fonction des molécules.

Le logiciel Hydras OTTC fournit avec la station d'acquisition de données ne permettant de ne représenter que deux abscisses, les graphes des résultats représentant la pluviométrie, le débit et les concentrations en pesticides ont été réalisés par programmation sous $\mathrm{R}$.

\section{RÉSUltats}

\section{Composition des sols}

Le tableau 5 présente la composition du sol en fonction de l'évolution de la pente. Il y a des caractéristiques qui différencient les sols du haut de pente présentant un horizon de départ en argile et les sols du bas de pente présentant un horizon d'accumulation en argile. La profondeur des sols augmente en fonction de la pente. Ces sols peu épais et caillouteux témoignent d'une forte sensibilité à

\begin{tabular}{|c|c|c|c|c|c|c|c|c|}
\hline Sols & $\begin{array}{c}\text { Sables } \\
\text { grossiers } \\
2000-200 \mu \mathrm{m} \\
(\%)\end{array}$ & $\begin{array}{c}\text { Sables fins } \\
200-50 \mu \mathrm{m} \\
(\%)\end{array}$ & $\begin{array}{c}\text { Limons } \\
\text { grossiers } \\
20-50 \mu \mathrm{m}(\%)\end{array}$ & $\begin{array}{l}\text { Limons fins } \\
2-20 \mu \mathrm{m}(\%)\end{array}$ & $\begin{aligned} & \text { Argiles } \\
< & 2 \mu \mathrm{m}(\%)\end{aligned}$ & $\begin{array}{c}\text { Matières } \\
\text { organiques } \\
(\%)\end{array}$ & $\mathrm{pH}$ (water) & $\mathrm{CaCo}_{3}(\%)$ \\
\hline Tti & 16,1 & 16,7 & 18,2 & 22,5 & 23,9 & 2,5 & 7,3 & 0,1 \\
\hline TG & 17,6 & 17 & 18,8 & 21,4 & 22,7 & 2,5 & 7 & 0,1 \\
\hline Mti & 22,2 & 21,2 & 17,3 & 19,4 & 17,9 & 1,9 & 7,2 & 0,1 \\
\hline MG & 25,7 & 19,2 & 17,1 & 18,9 & 16,8 & 2,2 & 6,8 & 0,1 \\
\hline $\mathrm{DCw}$ & 20,3 & 22,2 & 18,3 & 17,4 & 20,1 & 1,6 & 7 & 0,1 \\
\hline DG & 18,3 & 23,3 & 19,1 & 17,7 & 19,9 & 1,6 & 7 & 0,1 \\
\hline fraction instable Tti & & 16,74 & 21,94 & 55,2 & 8,84 & & & 0,1 \\
\hline fraction instable TG & & 17,24 & 19,39 & 54,02 & 9,35 & & & 0,1 \\
\hline $\begin{array}{l}\text { fraction instable } \\
D_{\text {DCw }}\end{array}$ & & 25,65 & 19,64 & 48,53 & 6,12 & & & 0,1 \\
\hline $\begin{array}{c}\text { fraction instable } \\
\text { DG }\end{array}$ & & 27,35 & 20,26 & 45,52 & 3,8 & & & 0,1 \\
\hline
\end{tabular}

TG et TTi : sols de haut de pente gazonnée (TG) et labouré (TTi), MG et MTi : milieu de pente enherbée (MG) et labouré (MTI) et DG et DCW : sol de bas de pente enherbé (DG) et chimiquement désherbé (DCW).

Tableau 5 : Caractéristiques des sols du site d'étude Characteristics of the soils in the studied area 
la sécheresse et de ce fait possèdent une réserve utile faible. Les sols de haut de pente présentent moins de sables $(32,8-34,6 \%$ contre 41,6-44,9\%) et plus de limons (40,7-40,2\% contre 35,7-36,8\%) et une plus grande teneur en matières organiques (MO : 2,5\% contre 1,6 et 2,2\%). On constate une diminution de la teneur en MO le long de la pente dans le bassin-versant avec peu ou pas de différence entre les parties enherbées et désherbées de chaque zone. Le long de cette topo-séquence il n'y a pas de variation significative de $\mathrm{pH}$ (=7, Tété, 2008), de même pour les teneurs en $\mathrm{CaCO}_{3}$, proches de zéro, car les sols sont développés sur des schistes et donc pauvres en $\mathrm{CaCO}_{3}$. Les formes de calcaires retrouvées proviennent essentiellement du chaulage effectué par les viticulteurs.

La fraction fine dite instable $(<250 \mu \mathrm{m})$ représentée par les particules de sol les moins stables qui sont généralement issues de la désagrégation des agrégats. Elle est principalement composée à $80 \%$ de limons fins et d'argiles. Comparée au sol de haut de pente, la fraction instable de bas de pente est plus riche en sables $(26-17 \%)$ et plus pauvre en limons et en argiles (65\% de limon et $6 \%$ d'argiles contre $74 \%$ et $9 \%$ d'argiles). La quantité d'agrégats stables à la désagrégation par l'eau est faible pour les sols travaillés (désherbés mécaniquement par labour) comparativement aux sols restés enherbés (figure 6 - planche V). On constate également pour le haut de pente que la fraction instable augmente inversement à la quantité d'agrégats stables (figure 7).

\section{Année hydrologique 2012}

L'année 2012 fût particulièrement excédentaire en précipitations à la station de mesures par rapport à la normale et ce comparativement à l'année précédente, 2011, plutôt déficitaire (figure 4). Les mois les plus excédentaires ont été avril et octobre 2012 et ont donc fait l'objet de plusieurs campagnes d'échantillonnages des eaux de ruissellement. Les coefficients de ruissellement en 2012 ont atteint des records avec une valeur obtenue de $57 \%$ pour des

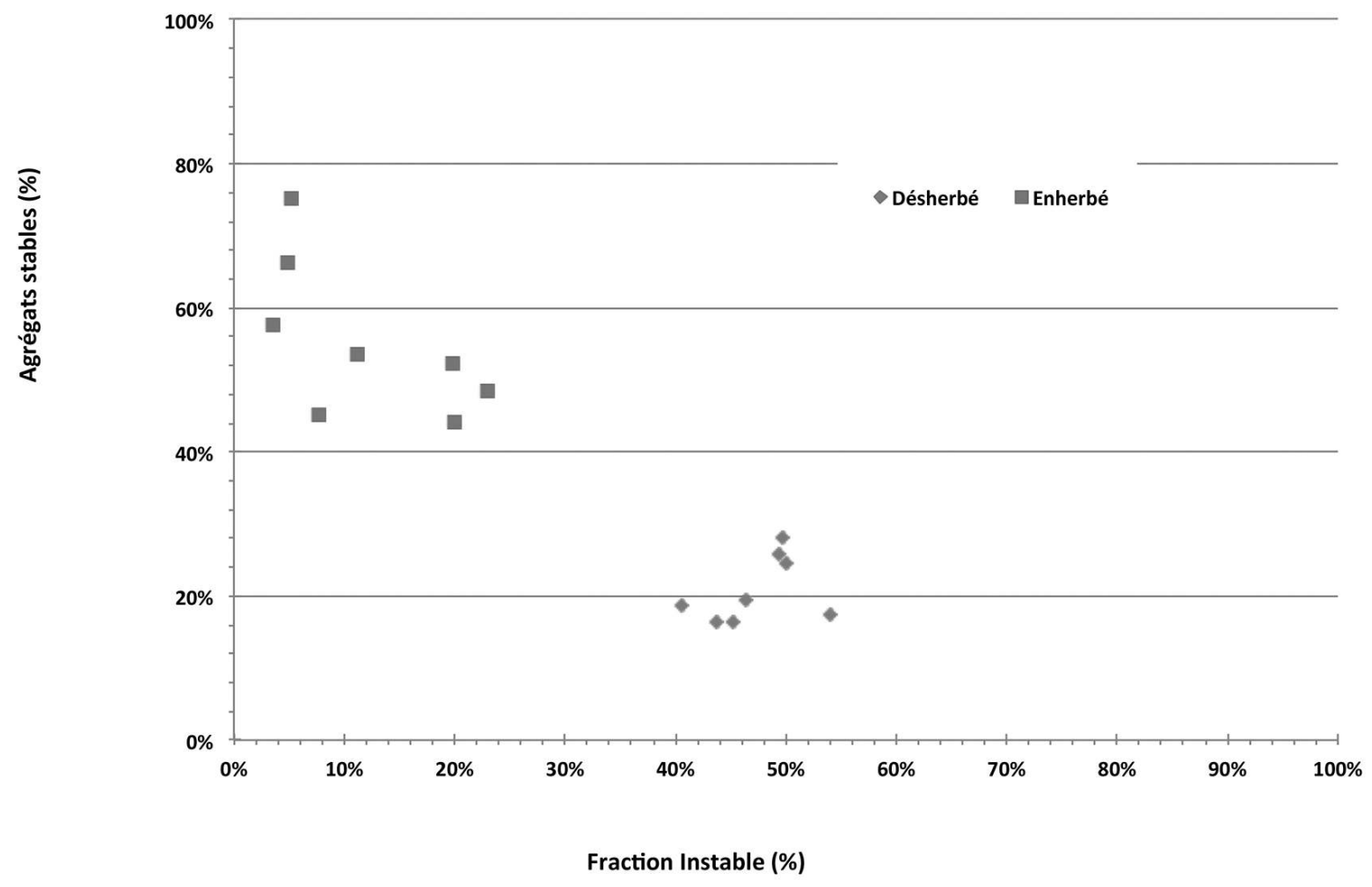

Figure 7 : Mesure de la stabilité structurale du sol (teneur en agrégats stables et teneur en fraction instable exprimée en pourcents) pour le haut de pente pour un sol enherbé (E) et désherbé mécaniquement $(\mathrm{D})$

Measurement of soil structural stability (content of stable aggregates and content of unstable soil fraction expressed as a percentage) of the upper slope for grassed soil (E) and for soil weeded mechanically (D) 
sols restés complètement saturés en eau pendant plusieurs jours en octobre.

\section{Ruissellement et érosion}

En 2012, les concentrations en MES sont comprises, en moyenne par crue, entre 80 et $300 \mathrm{mg} / \mathrm{L}$ (figure 8). Des événements pluvieux plus intenses ont cependant généré des concentrations plus importantes. Ainsi, en octobre 2012, les concentrations atteignent $1500 \mathrm{mg} / \mathrm{L}$. Ces concentrations en MES maximales se situent lors du pic de crue. À cette concentration s'associe une forte érosion puisqu'elle représente un transfert d'environ $7 \mathrm{~kg}$ de MES en 4 minutes.

On constate sur la figure 9 (planche $\mathbf{V}$ ) que les sols enherbés ont produit moins de fraction instable que les sols désherbés. La fraction instable est composée ici essentiellement par des limons fins et argiles. Il y a une faible teneur en argiles pour faire office de ciment et composer les agrégats stables.

\section{Pics de pesticides}

Le suivi des concentrations en pesticides véhiculés par ruissellement est réalisé lors des crues et présenté ici pour l'année 2012. Les quantités de pesticides appliquées sur les parcelles en vignes ont en fait été moins importantes que pour les années précédentes et notamment par rapport à 2011 (tableau 5). Le viticulteur de la parcelle B (figure 4) est en conversion en agriculture biologique. On constate que les concentrations maximales dans les eaux de ruissellement décroissent dans le temps plus les événements pluvieux sont éloignés des dates d'application du produit présentées dans le tableau 6. Ceci est généralement observé dans la littérature (Coupe et al., 2012). Toutefois, cela semble moins simple pour le couple glyphosate/ AMPA. Pour l'année 2012, la gamme des concentrations de glyphosate (figure 9 - planche $\mathbf{V}$ ) n'excède pas le seuil de $1 \mu \mathrm{g} / \mathrm{L}$ mais il reste présent dans toutes les campagnes d'échantillonnages et ce malgré une dégradation du glyphosate théorique durant l'année puisque la demi-vie aux champs se situe autour de 130 jours (tableau 7). La concentration en AMPA est par contre toujours supérieure à celle du glyphosate et atteint $7 \mu \mathrm{g} / \mathrm{L}$. Les pics de concentrations en AMPA et en glyphosate lors d'une crue se situent tous aux maximums de débit, mais dans des rapports réguliers. Il est à noter également que le glyphosate est, pour ce bassinversant, le premier pesticide appliqué en terme de quantité (tableau 6).

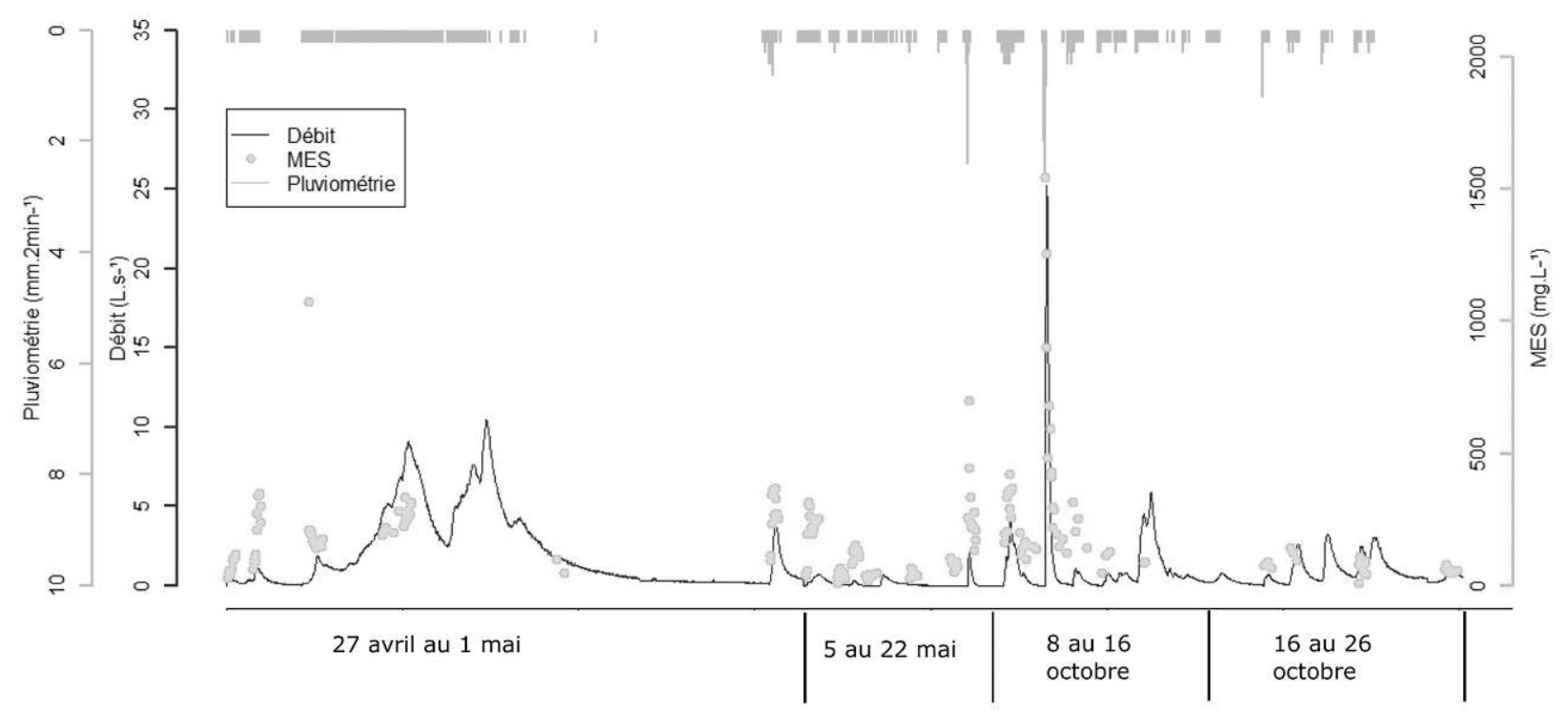

Figure 8: Pluviométrie, débit et concentrations en matières en suspension (MES) des échantillons d'eau de ruissellement prélevés lors d'événements pluvieux de l'année hydrologique 2012 hydrological year

Rainfall, flow and suspended matter concentrations (MES) from samples of surface run-off collected during events of the 2012 
Isabelle LA JeUnESSE et al. - NOROIS n 235 (2015/2) p. 67-86

\begin{tabular}{|c|c|c|c|c|}
\hline Molécules appliquées & $\begin{array}{c}\text { Quantités en } 2011 \\
\text { (g/2,2 ha) }\end{array}$ & Dates d'applications 2011 & $\begin{array}{c}\text { Quantités en } 2012 \\
\text { (g/2,2 ha) }\end{array}$ & Dates d'applications 2012 \\
\hline Glyphosate & 1291 & 25 mars et 4 avril & 2144 & 11 mai et 19 juin \\
\hline Dithianon & 415 & 7 juin & 498 & 2 juillet \\
\hline Diméthomorphe & 178 & 7 juin & 231 & 2 juillet et 25 juillet \\
\hline Zoxamide & 118 & 6 mai & 0 & \\
\hline Fluopicolide & 105 & 6 mai & 0 & \\
\hline Kresoxim méthyl & 79 & 11 mai & 0 & \\
\hline Trifloxystrobine & 60 & 18 mai & 0 & \\
\hline Tébuconazole & 54 & 6 mai & 0 & \\
\hline Quinoxyfène & 43 & 10 juin & 99 & 2 juillet \\
\hline Iprovalicarbe & 0 & & 90 & 25 juillet \\
\hline Spinosad & 0 & & 46 & 5 juin \\
\hline Tétraconazole & 40 & 26 avril et 7 juin & 40 & 3 mai et 8 juin \\
\hline Triadiménol & 18 & 6 mai & 0 & \\
\hline Penoxsulam & 3 & 4 avril & 0 & \\
\hline
\end{tabular}

Tableau 6 : Quantités de matières actives appliquées sur le bassin-versant (de 2,2 hectares) et dates d'applications en 2011 et en 2012 Quantities of active substances applied in the catchment and dates of application in 2011 and 2012

Seules 7 des molécules appliquées (tableau 6) sont en fait retrouvées dans les eaux de ruissellement (figure 9 - planche V). Cela signifie que les deux non retrouvées sont soient totalement dégradées (en dessous du seuil de quantification), hypothèse la plus probable face aux durées de dégradation de la moitié de la quantité du produit (tableau 7), soit adsorbées, soit lessivées ou encore ingérées par des organismes biologiques. Le diméthomorphe, le fluopicolide et le tétraconazole, tous trois des fongicides, n'avaient pas été appliqués avant l'épisode du $1^{\mathrm{er}}$ mai 2012. Leurs dernières applications datent en fait du mois de juin 2011. Ceci montre une rémanence des molécules dans le sol au moins supérieure à un an. En effet, ces trois molécules sont connues pour présenter des durées de demi-vie assez longues : 2 à 3 mois pour le diméthomorphe, 2 à 6 mois pour le fluopicolide et de plus d'un an pour le tétraconazole.

Les pesticides, même ceux de la phase dissoute de l'eau ruisselée, proviennent majoritairement de la quantité de sol érodé. Ce phénomène est confirmé pour les molécules dont les concentrations évoluent avec les concentrations en MES dans les eaux, généralement elles aussi corrélées au débit.

\section{Discussion}

Aux événements pluvieux les plus intenses correspondent les événements les plus chargés en MES et les plus concentrés en pesticides avec également le plus grand nombre de molécules détectées. Ainsi, les événements les plus intenses sont ceux qui remobilisent le plus les pesticides, supposés adsorbés et non dégradés dans les sols, conjointement à une forte érosion des sols. Au-delà de la nécessité de réaliser une étude d'adsorption pour valider ces hypothèses, ce constat pose un double enjeu pour les pratiques agricoles dans les parcelles viticoles.

En effet, dans le contexte de sensibilisation aux transferts de pesticides des parcelles viticoles aux milieux aquatiques, un inter-rang sur deux est laissé enherbé toute l'année et ce malgré une compétition forte en eau entre la vigne et l'herbe déplorée par les viticulteurs. Pour le viticulteur de la parcelle B (Figure 4), l'inter-rang est désherbé mécaniquement par labour depuis 2011 en raison de sa conversion en agriculture biologique. Il est désherbé chimiquement pour la parcelle du viticulteur A. Les chemins ne sont eux jamais désherbés. De nombreux auteurs ont constaté que le labour détruit la structure des sols (Le Bissonnais and Singer, 1993; Amézketa 1999; Barthès and Roose, 2002 and Ramos et al., 2003). Cette alternance de sol travaillé et non travaillé sur le bassin-versant pourrait diminuer l'infiltration et augmenter la dispersion de limons et d'argiles. Ainsi, si le désherbage mécanique évite l'utilisation de désherbant dont le glyphosate, il contribue à augmenter la sensibilisation du sol à 


\begin{tabular}{|c|c|c|c|c|c|c|c|c|c|}
\hline $\begin{array}{l}\text { Substances } \\
\text { Actives }\end{array}$ & Famille & Action & Solubilité & $\begin{array}{l}\text { Volatilisa- } \\
\text { tion }\end{array}$ & Affinité MO & Mobilité & $\begin{array}{c}\text { Potentiel } \\
\text { transport } \\
\text { colloïdal }\end{array}$ & $\begin{array}{c}\text { DT50 (jours } \\
\text { en labora- } \\
\text { toire ou aux } \\
\text { champs) }\end{array}$ & Persistance \\
\hline Diméthomorphe & Fongicide & mildiou & faible & non volatile & faible & modérée & moyen & 41 à 96 J & modérée \\
\hline Dithianon & Fongicide & mildiou & faible & non volatile & élevée & modérée & moyen & 10 à $35 \mathrm{~J}$ & modérée \\
\hline Fluopicolide & Fongicide & mildiou & faible & non volatile & modérée & modérée & moyen & 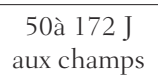 & persistant \\
\hline Glyphosate & Herbicide & $\begin{array}{l}\text { pré-post } \\
\text { levée }\end{array}$ & élevée & non volatile & faible & $\begin{array}{l}\text { très } \\
\text { modérée }\end{array}$ & moyen & $\begin{array}{c}3 \text { à } 40 \mathrm{~J} \\
\text { en labo, } 7 \\
\text { à } 130 \text { aux } \\
\text { champs }\end{array}$ & modérée \\
\hline Iprovalicarbe & Fongicide & mildiou & faible & non volatile & élevée & modérée & faible & 20 à $30 \mathrm{~J}$ & $\begin{array}{c}\text { non } \\
\text { persistant }\end{array}$ \\
\hline Kresoxim méthyl & Fongicide & oïdium & faible & non volatile & élevée & modérée & faible & $1,3 \mathrm{~J}$ & $\begin{array}{c}\text { non } \\
\text { persistant }\end{array}$ \\
\hline Penoxsulame & Herbicide & $\begin{array}{l}\text { pré-post } \\
\text { levée }\end{array}$ & modérée & non volatile & faible & mobile & faible & $22 a ̀ 58$ j & modérée \\
\hline Quinoxyfène & Fongicide & ö̈dium & faible & non volatile & élevée & non mobile & élevé & $>224 \mathrm{~J}$ & $\begin{array}{c}\text { très } \\
\text { persistant }\end{array}$ \\
\hline Spinosad & Insecticide & $\begin{array}{c}\text { mouche/ } \\
\text { chenilles/ } \\
\text { tordeuse de } \\
\text { la grappe }\end{array}$ & modérée & non volatile & élevée & - & - & $13 \mathrm{~J}$ & $\begin{array}{c}\text { non } \\
\text { persistant }\end{array}$ \\
\hline Tébuconazole & Fongicide & mildiou & faible & non volatile & élevée & $\begin{array}{c}\text { très } \\
\text { modérée }\end{array}$ & moyen & $>365 \mathrm{~J}$ & $\begin{array}{c}\text { très } \\
\text { persistant }\end{array}$ \\
\hline Tétraconazole & Fongicide & $\begin{array}{l}\text { mildiou/ } \\
\text { rougeot/ } \\
\text { blackrot }\end{array}$ & modérée & non volatile & élevée & $\begin{array}{c}\text { très } \\
\text { modérée }\end{array}$ & élevé & $\begin{array}{l}430 \mathrm{~J} \text { aux } \\
\text { champs }\end{array}$ & $\begin{array}{c}\text { très } \\
\text { persistant }\end{array}$ \\
\hline Triadimenol & Fongicide & ö̈dium & modérée & non volatile & élevée & $\begin{array}{l}\text { très } \\
\text { modérée }\end{array}$ & moyen & $\begin{array}{l}23 \text { à } 127 \mathrm{~J} \\
\text { en labo, } 6 \mathrm{~J} \\
\text { aux champs }\end{array}$ & persistant \\
\hline Trifloxystrobine & Fongicide & $\begin{array}{l}\text { mildiou/ } \\
\text { rougeot/ } \\
\text { blackrot }\end{array}$ & faible & non volatile & élevée & $\begin{array}{l}\text { très } \\
\text { modérée }\end{array}$ & moyen & $\begin{array}{l}2 \text { à } 12 \mathrm{~J} \text { aux } \\
\text { champ }\end{array}$ & $\begin{array}{c}\text { non } \\
\text { persistant }\end{array}$ \\
\hline Zoxamide & Fongicide & $\begin{array}{l}\text { mildiou/ } \\
\text { excoriose }\end{array}$ & faible & non volatile & élevée & $\begin{array}{c}\text { très } \\
\text { modérée }\end{array}$ & élevé & $\begin{array}{l}\text { 2à } 10 \mathrm{~J} \text { aux } \\
\text { champs }\end{array}$ & $\begin{array}{c}\text { non } \\
\text { persistant }\end{array}$ \\
\hline
\end{tabular}

Tableau 7 : Caractéristiques des molécules actives appliquées sur le site d'étude en 2012 (ACTA, AGRITOX 2014, PPDB 2015) Characteristics of active substance applied in the studied area in 2012

l'érosion lors d'une période qui suit le travail du sol. Toutefois, le travail du sol évite le compactage et augmente l'infiltration par augmentation de la porosité (Amézketa, 1999).

L'année 2012 a été une année exceptionnellement humide. Il y a donc eu potentiellement de forts effets de dilution. Les traitements, bien que nombreux en raison de la forte pression à l'oïdium et au mildiou, ont certes engagé des quantités de pesticides supérieures à 2011 mais pondérées par le fait qu'un des viticulteurs ait traité uniquement à la bouillie bordelaise sur toute la saison. Aussi, en l'état actuel des pratiques des viticulteurs, ce bassin-versant répond largement aux objectifs du plan
Ecophyto 2018 puisque les quantités appliquées ont été divisées par plus de $50 \%$. Ainsi, les concentrations en pesticides dans les eaux de ruissellement n'ont pas été très élevées en 2012 comparativement à d'autres années suivies sur ce site. Toutefois, on retrouve des molécules qui ont été appliquées plus d'un an auparavant et qui auraient dû être dégradées au vu des valeurs de leur demi-vie. Peut-être cette longue saturation des sols en eau a finalement permis la désorption de molécules présentes depuis des traitements bien antérieurs, hypothèse à laquelle seul un marquage radioactif pourrait répondre. La question de la rémanence dans les sols reste ainsi entière pour certaines molécules appliquées. 
En ce qui concerne les mécanismes de transfert, les plus fortes concentrations en pesticides correspondent toujours ici aux concentrations en MES les plus importantes. Ceci laisse à penser que les événements pluvieux les plus intenses et donc les plus érosifs sont susceptibles d'exporter plus de pesticides. Or l'érosion engage majoritairement la fraction instable des sols qui compose les MES. Il est cependant fort probable que de ce sol enherbé, plus stable, les particules se détachent moins. Ainsi son potentiel de désorption serait moindre qu'un sol nu.

\section{Conclusion}

La présente étude, qui place les modalités de transfert des pesticides au cœur des enjeux de restauration de la qualité des eaux du Layon portée par le SAGE Layon-Aubance-Louet conforte, pour les caractéristiques de ce bassin-versant, la bibliographie existante sur les modalités de transferts des pesticides en territoire viticole.

Tout d'abord, l'état d'enherbement du bassin-versant ne permet pas d'éviter tout transfert de pesticides dans ce petit bassin-versant viticole. Il est néanmoins important de rappeler que ce site est particulièrement pentu, surtout dans la partie la plus proche de l'exutoire, et que l'important phénomène d'érosion contribue à ce constat. Toutefois, cet enherbement contribue à une meilleure stabilité structurale et donc à limiter l'érosion. Or, l'étude montre que les pics de concentrations en pesticides coïncident systématiquement, et ce pour toutes les molécules suivies, aux pics de matières en suspension, autrement dit au pic d'érosion. Ainsi, l'enherbement, en limitant l'érosion, contribue à limiter les transferts des molécules adsorbées sur les particules fines du sol constituant les matières en suspension lors des phénomènes d'érosion et de ruissellement.

Également, l'étude montre que les concentrations en pesticides relevées dans les eaux de ruissellement sur l'année 2012 se situent toujours bien au-dessus de $1 \mu \mathrm{g} / \mathrm{L}$. Pourtant, en 2012, un des deux viticulteurs s'était déjà converti depuis 1 an à l'agriculture biologique, n'appliquant plus de molécules de synthèse recherchées dans les analyses.

Si les concentrations en pesticides dans les échantillons des eaux ruisselées diminuent lorsque l'on s'éloigne de la période de traitement, le suivi sur l'année hydrologique 2012 a montré que des pes- ticides sont toujours quantifiés, avec une présence systématique du glyphosate et de l'AMPA. Pourtant, cet herbicide n'est appliqué qu'une seule fois dans l'année et a une demi-vie bien inférieure à une année. Ainsi, les quantités de glyphosate stockées dans les sols semblent importantes, et se remobilisent au cours de la succession d'événements pluvieux. Ainsi, seul un suivi préalable de la teneur dans les sols permettrait de pouvoir réaliser un bilan des quantités transférées en fonction des quantités appliquées.

En ce qui concerne les objectifs du SAGE LayonAubance-Louet, l'atteinte d'un niveau de concentrations en pesticides de $1 \mu \mathrm{g} / \mathrm{L}$ à l'horizon 2018 semble très ambitieux au regard des pratiques actuelles. En effet, le bassin-versant étudié, alors que la moitié de sa surface est aujourd'hui en agriculture biologique, génère toujours des eaux de ruissellement avec des concentrations en pesticides bien au-delà des objectifs fixés.

Enfin, l'étude montre que plus il pleut et surtout plus il pleut fort, plus on retrouve les pesticides appliqués précédemment voire même des années hydrologiques antérieures et avec des pics de concentrations qui suivent les pics de débit et de MES liées à l'érosion. Ainsi, l'effet de dilution, souvent avancé comme élément déterminant pour améliorer la qualité des eaux par opposition aux problématiques d'étiage, n'est pas constaté comme positif pour la qualité des eaux du Layon. L'état du sol et sa capacité de rétention semble être le facteur gouvernant les transferts de pesticides et donc à considérer pour lutter contre la contamination du Layon.

Cependant, face à l'efficacité insuffisante de l'enherbement partiel des inter-rangs de vignes, les méthodes de remédiation sont difficiles à trouver tant la diversité de comportement des molécules visà-vis du sol dépend en fait des caractéristiques du sol qui définissent non seulement la sensibilité du sol à l'érosion (désagrégation par l'eau) mais aussi l'affinité pour la molécule (phénomène d'adsorption/désorption). Notamment, l'affinité pour certaines composantes du sol dont les particules fines est un élément important dans le transfert, or cette affinité varie en fonction des caractéristiques des molécules actives. Des bureaux d'étude proposent d'ailleurs des programmes de sensibilisation au choix des molécules, en fonction de type de sol (et 
donc de la problématique adsorption/désorption) et ce par l'utilisation de modèles experts, qui semblent donc pertinents. Toutefois, le niveau de précision des connaissances sur la composition des sols à l'échelle du territoire, indépendamment du niveau de complexité des modèles utilisés, demeure un frein important au développement de l'utilisation de ces outils d'aide à la décision par les gestionnaires de bassins-versants.

Ainsi, quels que soient les efforts réalisés sur la diminution progressive des applications de pesticides dans les objectifs, aucun dispositif, sauf à ceinturer les bassins-versants pour capturer les ruissellements et les particules fines érodées avant que les eaux ne gagnent les cours d'eau, n'est envisageable à grande échelle pour limiter les transferts d'eaux de ruissellement, riches en pesticides, vers les cours d'eau récepteurs.

\section{Remerciements}

Les résultats présentés ici sont extraits d'une étude financée par le Conseil Régional des Pays-de-la-Loire, l'Agence de l'Eau Loire-Bretagne, FranceAgriMer, InterLoire et ayant également bénéficié du soutien de la profession viticole, de la mairie de Rochefort-surLoire et de l'université d'Angers dans le cadre notamment du Contrat Régional des Bassins Versants du Layon et de l'Aubance et du Contrat Territorial des bassins du Layon et de l'Aubance. Les auteurs remercient tous chaleureusement Stéphane Sourice, assistant ingénieur du laboratoire, pour son aide au bon déroulement du projet depuis ses balbutiements ainsi que tous les collègues et étudiants qui ont pu participer aux campagnes d'échantillonnages.

\section{Bibliographie}

Abdoul-Madjidi M.-A., 2008. Analyse statistique de la série de concentrations de pesticides dans le Layon, Mémoire de Master Ecologie, Université d'Angers, 60 p.

ACTA, AGRITOX 2014. Base de données sur les substances actives phytopharmaceutiques. [http://www.agritox.anses.fr].

Amézketa E., 1999. Soil Aggregate Stability: A Review, J. Sustain. Agric., 14, p. 83-151. doi :10.1300/J064v14n02_08.

Andresen L.C., Nothlev J., Kristensen K., Navntoft S., Johnsen I., 2012. The wild flora biodiversity in pesticide free bufferzones along old hedgerows, Journal of Environmental Biology, 33, p. 565-572.

Aparicio V.C., De Gerónimo E., Marino D., Primost J., Carriquiriborde P., Costa J.L., 2013. Environmental fate of glyphosate and aminomethylphosphonic acid in surface waters and soil of agricultural basins, Chemosphere 93, 1866-1873. doi :10.1016/j.chemosphere.2013.06.041.

Aubertot J.-N., Barbier J.-M, Carpentier A., Gril J.-J., Guichard L., Lucas P., Savary S., Savini I., Voltz M., 2005. Pesticides, agriculture et environnement. Réduire l'utilisation des pesticides et limiter leurs impacts environnementaux, Expertise scientifique collective, synthèse du rapport, INRA et Cemagref (France), 64 p.

Barthès B., Roose E., 2002. Aggregate stability as an indicator of soil susceptibility to runoff and erosion; validation at several levels, CATENA 47, p. 133-149. doi :10.1016/ S0341-8162(01)00180-1.

Barriuso E., Calvet R., 1992. Soil type and herbicides adsorption, Intern. J. Environ. Anal. Chem., 46, p. 117-128.

Barriuso E., Calvet R., Schiavon M., Soulas G., 1996. Les pesticides et les polluants organiques des sols - Transformations et dissipation, Étude et gestion des sols, 3(4), p. 279296.

Bassil K.L., Vakil C., Sanborn M., Cole D.C., Kaur J.S., Kerr K.J., 2007. Cancer health effects of pesticides, Canadian Family Physician 53.10, p. 1704-1711.

BENOÎT P., 1994. Rôle de la nature des matières organiques dans la stabilisation de résidus de polluants organiques dans les sols, Thèse Institut National Paris Grignon, 209 p.

Calvet R., Barriuso E., Bedos C., Benoit P., Charnay M.-P., Coquet Y., 2005. Les pesticides dans le sol. Conséquences agronomiques et environnementales, Paris, France Agricole, $635 \mathrm{p}$.

Contrat territorial des bassins du Layon et de l'Aubance 2011 2015, 2010, 128 p.

Coupe R.H., Kalkhoff S.J., Capel P.D, Grégoire C., 2012. Fate and transport of glyphosate and aminomethylphosphonic acid in surface waters of agricultural basins, Pest Management Science, 68/1, p. 16-30.

Damonte M., Torres Sánchez R.M., dos Santos Afonso M., 2007. Some aspects of the glyphosate adsorption on montmorillonite and its calcined form, Appl. Clay Sci. 36, p. 86-94. doi :10.1016/j.clay.2006.04.015.

Daouk S., Copin P.J., Rossi L., Chèvre N., Pfeifer H.R., 2013. Dynamics and environmental risk assessment of the herbicide glyphosate and its metabolite AMPA in a small vineyard river of the lake Geneva catchment, Environmental Toxicology and Chemistry, Vol. 32, n 9, p. 2035-2044.

Dur C., Gouy V., Calvet R., Bélamie R., Chaplain V., 1998. Influence of adsorption-desorption phenomena on runoff measured under controlled conditions, Comptes rendus de l'Académie des sciences, 327 (6), p. 404-411.

Fitzmaurice A.G., Rhodes S.L., Lulla A., Murphy N.P., Lam H.A., O'Donnell K.C., Barnhill L., Casida J.E., Cockburn M., Sagasti A., Stahl M.C., Maidment N.T., Ritz B., Bronstein J.M., 2013. Aldehyde dehydrogenase inhibi- 
tion as a pathogenic mechanism in Parkinson disease, Proc. Natl. Acad. Sci. USA 110(2), p. 636-641.

Gatignol C., Etienne J.-C., 2010. Office parlementaire des choix technologiques et scientifiques. Rapport sur pesticides et santé, $n^{\circ} 2463$ de l'Assemblée Nationale et n 421 du Sénat, 262 p.

GLASs R.L., 1987. Adsorption of glyphosate by soils and clay minerals, J. Agric. Food Chem, 35, p. 497-500. doi :10.1021/ jf00076a013.

Goyard S., 2014. Étude des dynamiques du ruissellement, de l'érosion et du transfert des pesticides dans un bassin-versant viticole : approche par modélisation, Mémoire de fin d'étude d'ingénieur de l'ENGEES, Université de Strasbourg, 79 p.

Guérin B., 2008. Méthode de délimitation d'un bassin-versant viticole. Mémoire de Master Écologie, université d'Angers, $60 \mathrm{p}$.

Imfeld G., Villeumier S., 2012. Measuring the effects of pesticides on bacterial communities in soil : a critical review, European Journal of Soil Biology, 49, p. 22-30.

Kemper W.D., Rosenau R.C., 1986. Aggregate stability and size distribution. In KLute A. (éd.), Methods of Soil Analysis, Part 1. Agronomy Monographs, vol.9. American Society of Agronomy, Madison, WI.

Kohler H. R., Triebskorn R., 2013. Wildlife Ecotoxicology of Pesticides: Can We Track Effects to the Population Level and Beyond?, Science, 341.6147, p. 759-765.

La Jeunesse I., Jadas-Hécart A., Communal P.-Y., Duchesne J., Landry D., Sourice S., Moguedet G., Picard F., BalLouche A., Vitrai V., 2010. Pesticides en viticulture et qualité des eaux. Une réglementation face à une culture à haut risque de transfert, Revue des oenologues, vol. 137, p. 1-5.

Le Bissonnais Y., Le Souder C., 1995. Mesurer la stabilité structurale des sols pour évaluer leur sensibilité à la battance et à l'érosion, Étude et Gestion des Sols, 2/1, p. 43-56.

Le Bissonnais Y., Singer M.J., 1993. Seal formation, runoff and interrill erosion from seventeen California soils, Soil Sci. Soc. Am. J. 57, p. 224-229.

Le Bissonnais Y., 1996. Aggregate stability and assessment of soil crustability and erodibility: I. Theory and methodology. European Journal of Soil Science, 47, p. 425-437.

MEDDE, 2015. Observation et statistiques. [http://www.statistiques.developpement-durable.gouv.fr/lessentiel/ar/246/211/ contamination-globale-cours-deau-pesticides. $h \mathrm{tml}$ ].

Miguens T., Leiros M.C., Gil-Sotres F., Trasar-Cepeda C., 2007. Biochemical properties of vineyard soils in Galicia, Spain, Science of The Total Environment, 378, p. 218-222.

Mostafalou S., Abdollahi M., 2013. Pesticides and Human Chronic Diseases : Evidences, Mechanisms, and Perspectives, Toxicology and Applied Pharmacology 268.2, p. 157-177.

Oliver D.P., Kookana R.S., Anderson J.S., Cox J., Waller N., Sмiтн L., 2012. The off-site transport of pesticide loads from two land uses in relation to hydrological events in the Mt. Lofty Ranges, South Australia, Agric. Water Manag., 106, p. $70-77$.

ORP, 2015. Observatoire des Résidus de Pesticides [http://www. observatoire-pesticides.gouv.fr/].

Panuska J.C., Karthikeya, K.G., Miller P.S., 2008. Impact of surface roughness and crusting on particle size distribution of edge-of-field sediments, Geoderma 145, 315-324.

Petit K., 2014. La banque nationale des ventes réalisées par les distributeurs de produits phytosanitaires (BNV-D), ONEMA, $12 \mathrm{p}$.

PPDB, 2015. Pesticide Properties DataBase de l'Université d'Hertfordshire. [http://sitem.herts.ac.uk/aeru/ppdb/].

Rabiet M., Margoum C., Gouy V., Carluer N., Coquery M., 2010. Assessing pesticide concentrations and fluxes in the stream of a small vineyard catchment - Effect of sampling frequency, Environmental Pollution, 158, p. 737-748.

Rakotoarisoa M., Fleurant C., Аmiot A., Ballouche A., Communal P.-Y., Jadas-Hécart A., La Jeunesse I., Landry D., Razakamanana T., 2014. Un système multi-agents pour la modélisation des écoulements de surface sur un petit bassin-versant viticole du Layon, Revue Internationale de Géomatique, 24/3 : 307-333. doi :10.3166/rig.24, p. 307-333.

Ramos M.C., Nacci S., Pla I., 2003. Effect of raindrop impact and its relationship with aggregate stability to different disaggregation forces. CATENA 53, 365-376. doi :10.1016/ S0341-8162(03)00086-9.

SAGE, 2004. Document principal validé par la CLE, 110 p.

SAGE, 2013. État des masses du bassin en 2011, [www.eaulayon-aubance. $f r]$.

SIGES Pays-de-la-Loire, 2015 [http://sigespal.brgm.fr/spip. php?article72].

Saygina S.D., Cornelisb W.M., Erpula G., Gabriels D., 2012. Comparison of different aggregate stability approaches for loamy sand soils, Applied soil Ecology, 54, p. 1-6.

Schwarzenbach R.P., Escher B.I., Fenner K., Hofstetter T.B., Johnson C.A., Gunten U. von, Wehrli B., 2006. The Challenge of Micropollutants in Aquatic Systems. Science 313, p. 1072-1077.

TÉTÉ E.K., 2008. Etude de la répartition spatiale du pH du phosphore et du cuivre dans le sol Application sur un bassin-versant viticole de Rochefort-sur-Loire 49. Mémoire de Master Écologie, Université d'Angers, 47 p.

Vörösmarty C.J., McIntyre P.B., Gessner M.O., Dudgeon D., Prusevich A., Green P., Glidden S., Bunn S.E., Sullivan C.A., Liermann C.R., Davies P.M., 2010. Global threats to human water security and river biodiversity, Nature 468 (7321), p. 334.

WajDZik A., 2008. Trop de pesticides dans les eaux du Layon, Ouest-France, 15 mai, nº 19364. 


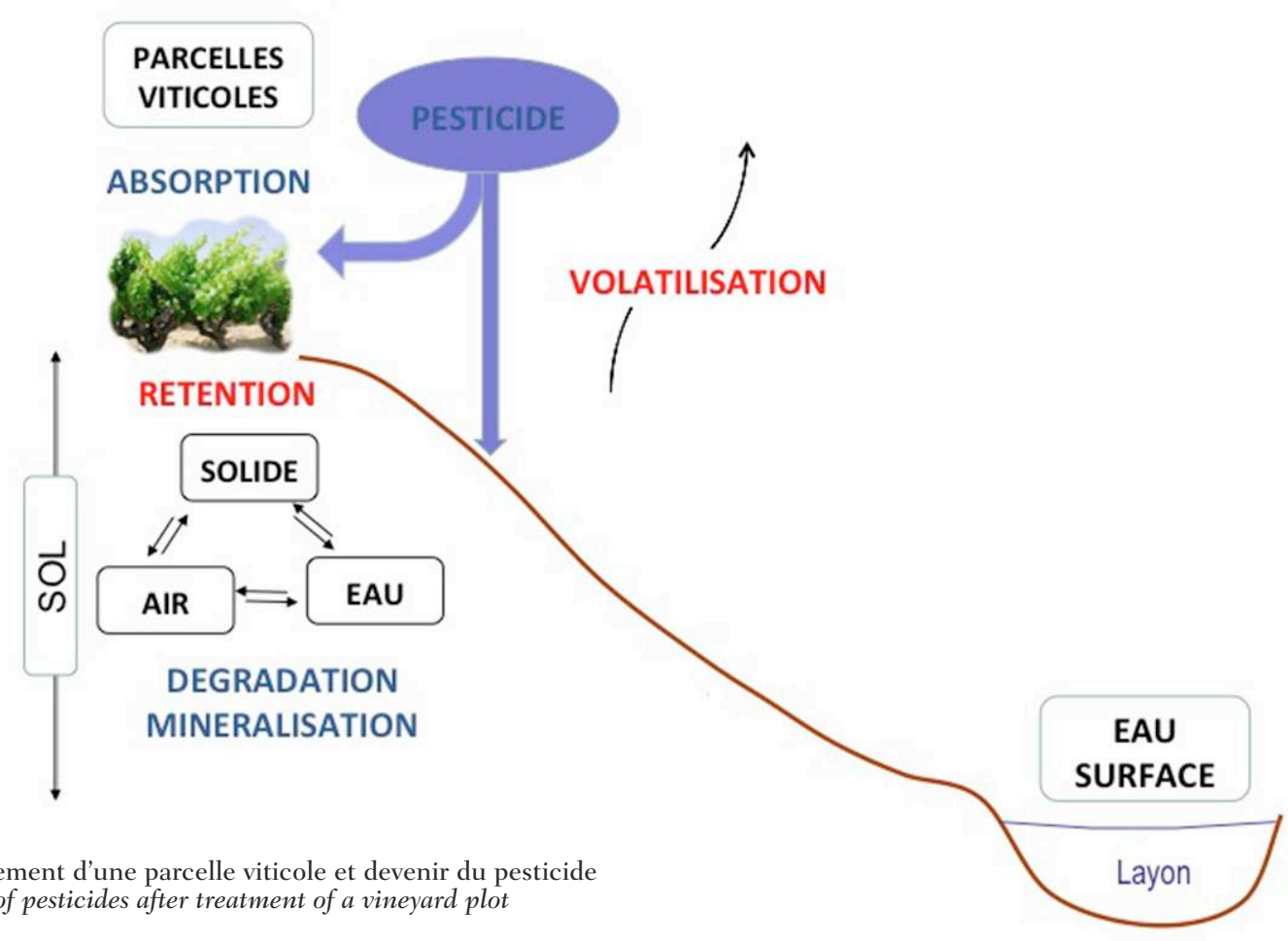

Figure 1: Traitement d'une parcelle viticole et devenir du pesticide Fate of pesticides after treatment of a vineyard plot

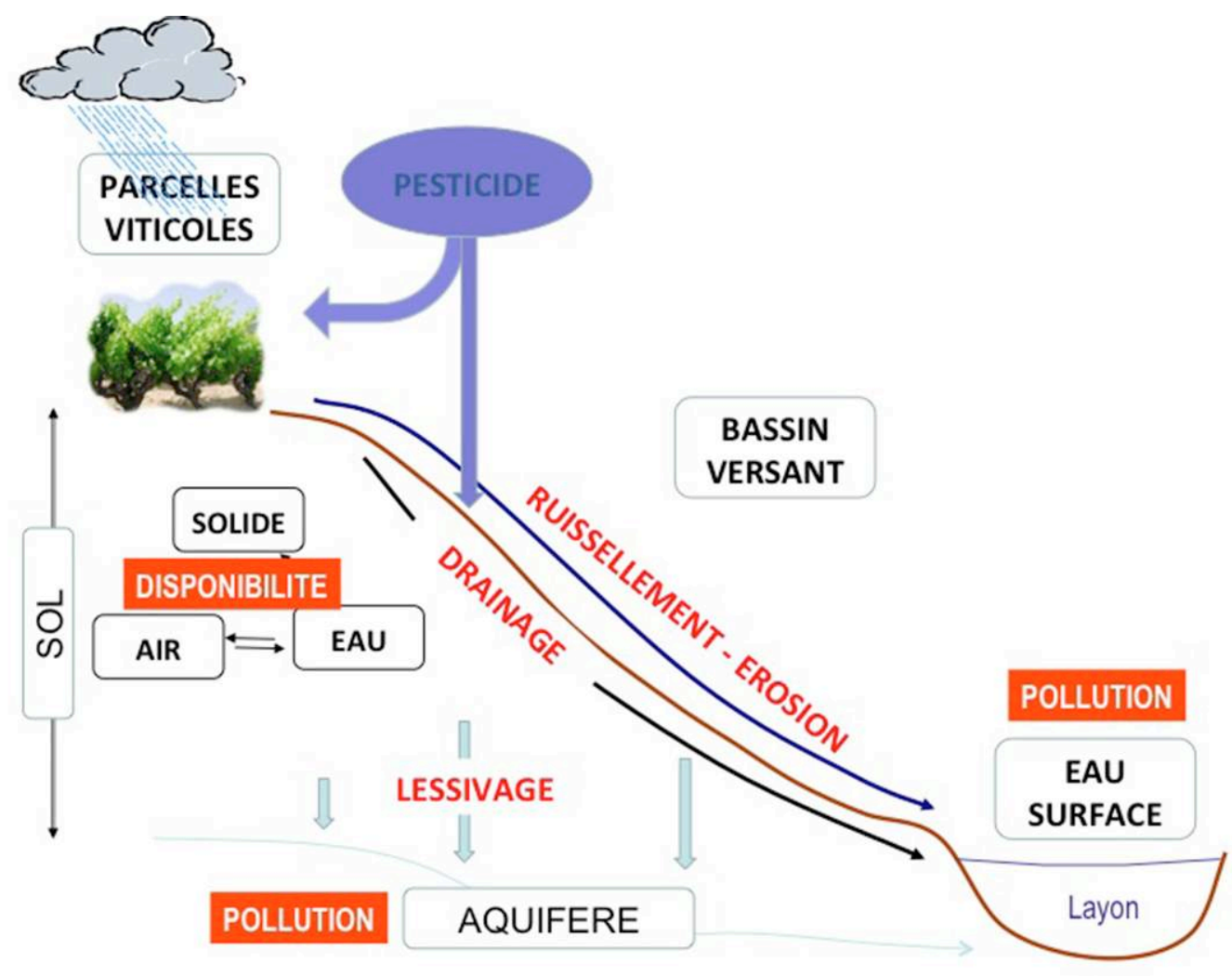

Figure 2 : Transfert du pesticide lors d'un événement pluvieux Transfer of pesticide during rainfall events 
Figure 6 : Cartographie du système de pente utilisée pour les prélèvements d'échantillons de sol. Les ruptures de pente définissent une topo-séquence composée de 3 zones : haut de pente $(\mathrm{H})$, milieu de pente $(\mathrm{M})$ et bas de pente $(\mathrm{B})$

Mapping slopes system for soil sampling. Slope failures define a topography sequence consisting of 3 areas: top of the slope $(H)$, mid-slope $(M)$ and down slope $(B)$
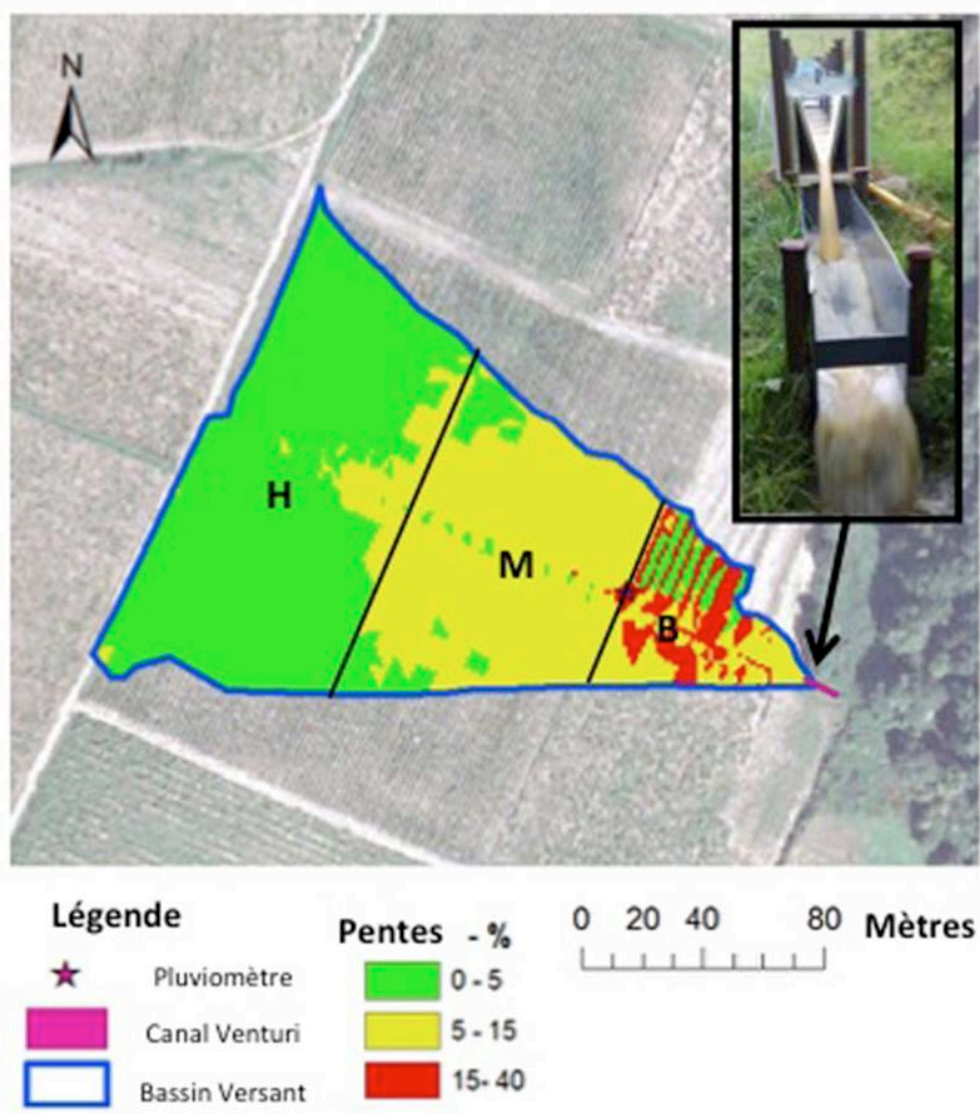

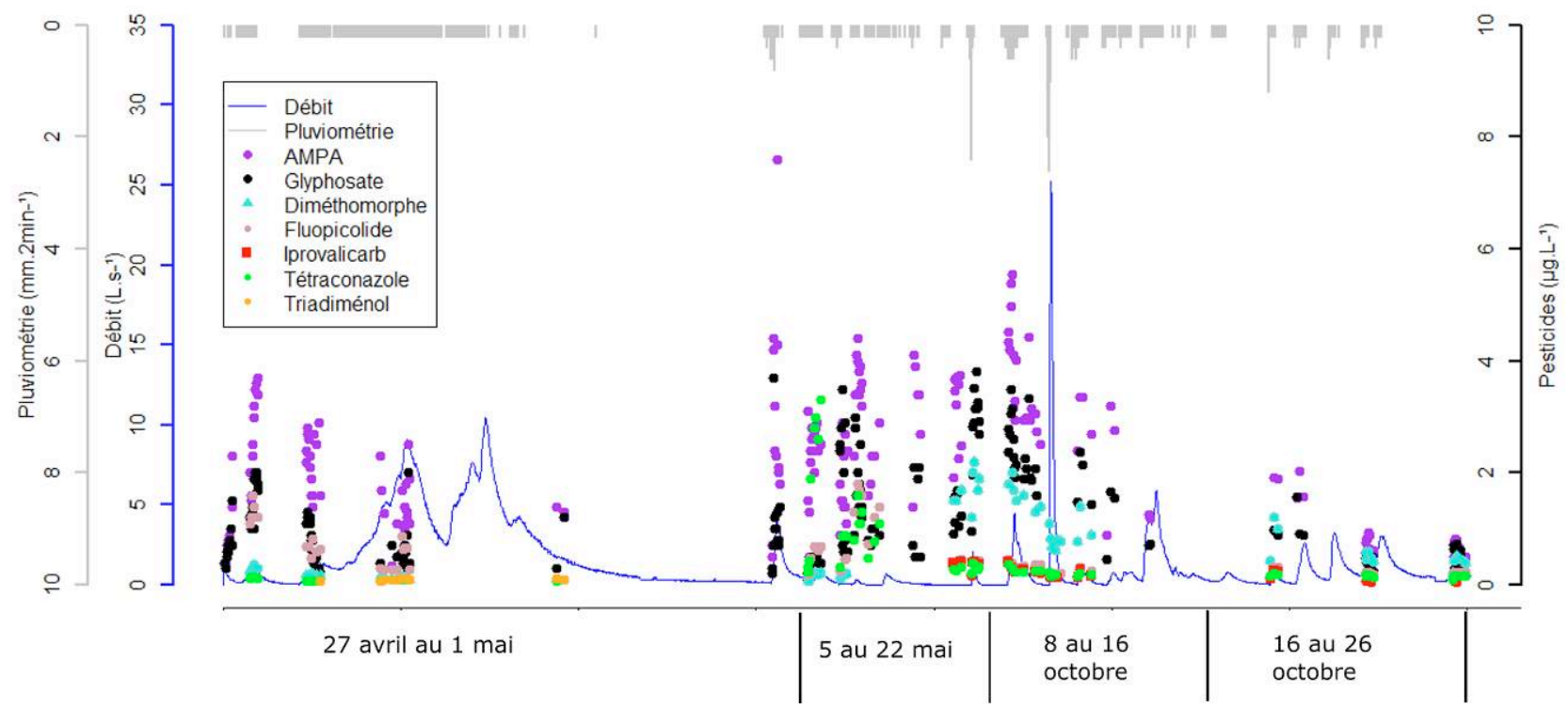

Figure 9 : Pluviométrie, débit et concentrations en pesticides des échantillons d'eau de ruissellement prélevés lors d'événements pluvieux de l'année hydrologique 2012

Rainfall, flow and pesticide concentrations from samples of surface run-off collected during events 